\title{
Novel string field theory with also negative energy constituents/objects gives Veneziano amplitude
}

\author{
H.B. Nielsen ${ }^{a}$ and M. Ninomiya ${ }^{b}$ \\ ${ }^{a}$ Niels Bohr Institute, University of Copenhagen, \\ 17 Belgdamsvej, DK 2100 Denmark \\ ${ }^{b}$ Advanced Mathematical Institute, \\ Osaka City University, 3-3-138 Sugimoto, Sumiyashi-ku 558-8585, Japan \\ E-mail: hbech@nbi.dk, msninomiya@gmail.com
}

Abstract: We have proposed a new type of string field theory. The main point of the present article is to cure some technical troubles: missing two out three terms in Veneziano amplitude. Our novel string field theory, describes a theory with many strings in terms of "objects", which are not exactly, but close to Charles Thorn's string bits. The new point is that the objects in terms of which the universe states are constructed, and which have an essentially 26-momentum variable called $J^{\mu}$, can have the energy $J^{0}$ be also negative as well as positive. We get a long way in deriving in this model the Veneziano model and obtain all the three terms needed for a four point amplitude. This result strongly indicates that our novel string field theory is indeed string theory.

KEywords: Bosonic Strings, Lattice Integrable Models, String Field Theory

ARXIV EPRINT: 1705.01739 


\section{Contents}

1 Introduction $\quad 2$

1.1 Our SFT model equivalent to string theory 3

1.2 Relation to Charles Thorn's string bits 3

1.3 Translation from strings to "cyclic chains" 4

1.4 The closed string 7

1.5 One open string would contribute say the slim closed curve contained in the net

1.6 We add one more - now wavy curve — open string contribution, a cyclic chain

1.7 Comments on our net of cyclic chains representing string via their right and left mover derivatives

$\begin{array}{lll}1.8 & \text { Further comments on novel string field theory model } & 10\end{array}$

$\begin{array}{ll}1.9 \text { The rough Dirac sea } & 11\end{array}$

1.10 Illustration of connection to the string $\quad 12$

2 The state of several strings $\quad 13$

$\begin{array}{ll}2.1 \text { A formalism of replacement of ghost } & 14\end{array}$

$\begin{array}{ll}2.2 \text { A trick of calculating the wave function } & 15\end{array}$

3 An encouraging step; the Veneziano model except for the integration $\begin{array}{ll}\text { weight } & 17\end{array}$

4 The classical approximation summary of "layers of existence degrees" 18

5 Main idea in calculating Veneziano amplitude 20

5.1 The simplest case of only positive $J^{+}=\frac{a \alpha^{\prime}}{2}$ (even) objects 23

5.2 The philosophy of counting 31

$6 \quad$ Evaluation of the integrand $\quad 32$

6.1 Evaluation of the integrand 32

6.2 What is required to finish Veneziano model? 34

7 Dominant term in $\mathbf{1}+\mathbf{2} \rightarrow \mathbf{3}+\mathbf{4}$ scattering $\quad 35$

7.1 Dominant term in $1+2 \rightarrow 3+4$ scattering 35

7.2 Problems in getting the weight of Veneziano integral easily 39

8 Conclusion and outlook $\quad 44$

A What is the rough Dirac sea? $\quad 46$

A.1 The idea of the rough Dirac sea 46

B Use of rough Dirac sea analogy for our novel string field theory background state 


\section{Introduction}

In order to describe a situation with several strings [1-10] you need a priori a string field theory $[11,12]$ — second quantization — of the of the strings like in Kaku and Kikkawa [1327] or Witten theory [28-38]. We should cite the pioneering paper hew by Mandelstam [39, 40]. Furthermore a seminal work on quantum string theory see [41]. But we have ourselves rather a description $[43,44]$, Starting in advance more similar to the string bit description of Charles Thorn et al. [45-50]) in which the state of an arbitrary number of strings is described by relating it to a state of a very large number of what we call "objects", and which have degrees of freedom like free particles.

The basic steps in writing in our formalism the second quantized state/Hilbert vector for a given set of strings are the following:

1. To every string construct the "cyclic chain(s)"- one for an open string and two for a closed one- in principle for each "classical state" by wiriting the developing (single string) state in terms of the splitting $X^{\mu}(\sigma, \tau)=X_{R}^{\mu}(\tau-\sigma)+X_{L}^{\mu}(\tau+\sigma)$. Then the curves presented by $\dot{X}_{R}^{\mu}(\tau-\sigma)$ parametrized by $\tau-\sigma$ and by $\dot{X}_{L}^{\mu}(\tau+\sigma)$ parametrized by $\tau+\sigma$ in $25+1$ dimensional Minkowski space-time. For the open string there is a trick that actually these two curves naturally continue each other into just one closed curve/just one closed cyclic chain.

2. Next these "cyclic chains" (from 1.) are discretized into small bits which we call objects. Notice that it is in the "light cone" variables $\tau_{R}=\tau-\sigma$ and $\tau_{L}=\tau+\sigma \underline{\text { we }}$ make discretization into a lattice of "objects" (Not like Thorn theory which discrete in $\sigma)$. Quantum mechanically some sophisticated trick is used only the even numbered lattice points $=$ objects (to avoid non-commutation of the object variables).

3. Next the many string state is represented by a Hilbert vector in an a priori free massless scalar particle quantum field theory by acting on a certain vacuum state $|0\rangle$ with a creation operator for each even (numbered) "object" in any one of the set of strings the state of which is to be constructed.

So the reader should see that we have made a correspondence which to any state of an arbitrary number of strings let correspond a Hilbert vector in a massless free scalar quantum field theory. I.e. we manage to make our novel string field theory become an ordinary quantum field theory!

From the previous works of ours it is important to remember: we develop a formalism for description of an arbitrary number of strings (String Field Theory) by means of a Hilbert space formally with "objects" that are more like particles (they are "bits" in a technically a little different way from Thorn's theory. We explain the difference in section 1.4.).

In terms of our "objects" (bits) the second quantized string world get totally static, scattering becomes a fake: the scattering amplitude becomes just the overlap of the initial with final states!

We get, after some technical procedure, the S-matrix $=$ the overlap between initial and final string states to be the Veneziano amplitude [51]. 


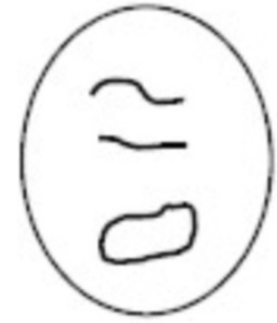

(a)

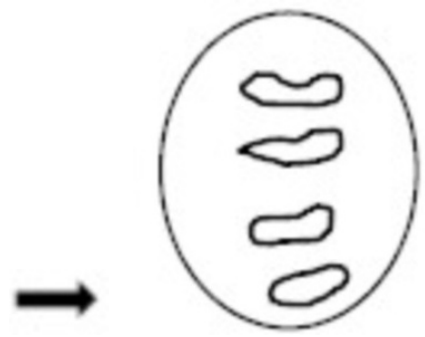

(b)

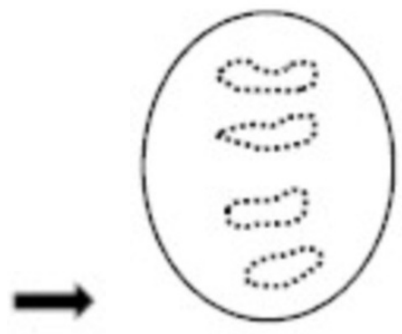

(c)

Figure 1. Open and closed strings (a) to our object formalism (b) and (c).

It is very crucial that the objects must be able to have energy of both signs.

So one piece of a cyclic chain can cancel another piece completely!

And thus pairs of compensating pieces of chains of objects may be phantazised where ever it may be.

"String" comes only in via the initial (and final state) conditions.

\subsection{Our SFT model equivalent to string theory}

The final SFT model(string field theory model) [42-44] of ours is described by the Fock space for massless non-interacting scalar bosons in $25+1$ dimensions. That is to say it is described by a Hilbert space, which is generated by a series of creation $a^{+}(\vec{p})$ and destruction $a(\vec{p})$ operators for scalar particels with 25 -momenta $\vec{p}$, which can act successively on a zero-particle state $|0\rangle$. That is to sey that the typical states in the Fock space - or the Hilbert space describing the world state - are

$$
a^{+}\left(\vec{p}_{1}\right) a^{+}\left(\vec{p}_{2}\right) \cdots a^{+}\left(\vec{p}_{n}\right)|0\rangle
$$

In the language, which we use, we call the scalar particles "even objects" and denote their momenta by $J^{\mu}$ instead of $p^{\mu}$ (well really we only consider the conjugate momenta for the transverse components $i=1,2, \ldots, 24$, i.e. $\left.J^{\mu}\right)$.

\subsection{Relation to Charles Thorn's string bits}

Our model/on string field theory is like they model for long considered prior to us by Charles Thorn also as a string bit theory in the sense that we discretize the strings. However, our way of discretizing deviates from the Thorn-version which makes the discretization string by discretizing the $\sigma$-variable. But in oar case rather by discretizing separately right mover and the left mover string like systems. That is to say we First write string variable $X^{\mu}$ as a sum of a left mover and a right mover part such that $X^{\mu}(\sigma, \tau)=X_{R}^{\mu}(\tau-\sigma)+X_{L}^{\mu}(\tau+\sigma)$ and then after that we perform the discretization by making what is essentially string bits but now for the variables $\sigma+\tau$ and $\sigma-\tau$ separately. we then to distinguish can call "objects" - instead after, we have split the solution into right and left mover and thus rather put into bits or now to distinguish objects the right-mover variable " $\tau-\sigma$ " or the left mover one " $\tau+\sigma$ ".

Actually Thorn has begun to do the same as we later at least for fermion modes. 

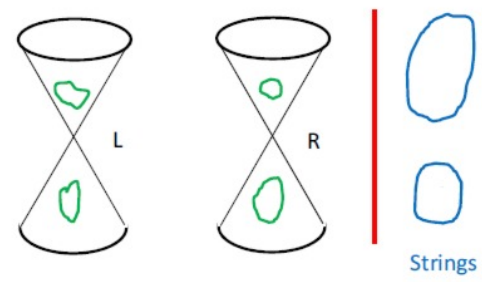

(a) For theory with only closed strings on the light cone.
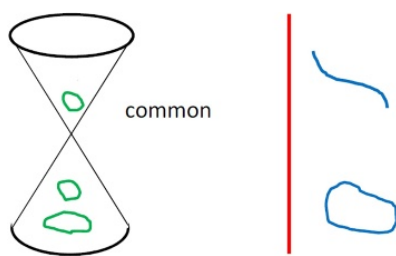

(b) For theories with also open strings

Figure 2. Chains of objects on the light cone in the $25+1$ dimensional Minkowski space. (a) is depicted on the closed strings in terms of the left and right movers; (b) The left and right movers of the open and closed strings are written together.

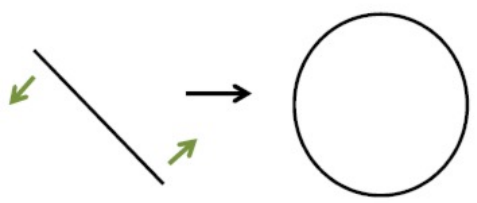

(a) Open string corresponds to topologically circular figure: the cyclic chain

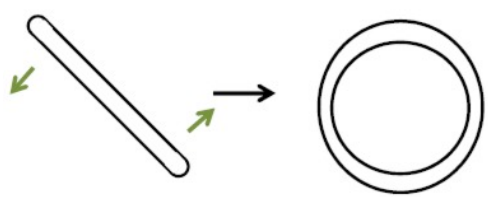

(b) Closed string corresponds to 2 different cyclic chain

Figure 3. Transition from strings to "cyclic chains".

\subsection{Translation from strings to "cyclic chains"}

As a very simple example of a classically described open strings, we can think of a string rotating as a stiff stick around its middle point. Say for simplicity that it is at rest and that we choose a gauge/a parametrization so that the energy is used to determine the parameter $\sigma$ along the string, so that an infinitesimal interval do in $\sigma$ just has the energy

$$
d E=d \sigma
$$

If $r$ denotes the distance from the middle point $C$ to the point with coordinate $\sigma$, and $R$ denotes half the geometrical length (in target space) of the (open) string, and $\omega$ the rotation rate, then the energy density at the point $\sigma$ is

$$
\frac{d \sigma}{d r}=\frac{d E}{d r}=\frac{1}{2 \pi \alpha^{\prime} \sqrt{1-v^{2}}}=\frac{1}{2 \pi \alpha^{\prime} \sqrt{1-\omega^{2} r^{2}}}
$$

Remembering

$$
\frac{d}{d(\omega r)} \arcsin (\omega r)=\frac{1}{\sqrt{1-(\omega r)^{2}}}
$$

thus

$$
\begin{aligned}
d \sigma & =\frac{d r}{2 \pi \alpha^{\prime}} \frac{d}{d(r \omega)} \arcsin (\omega r) \\
& =\frac{1}{2 \pi \alpha^{\prime} \omega} d \operatorname{arcsine}(\omega r)
\end{aligned}
$$


so that by integrating we may take the integration constant to get

$$
\sigma=\frac{1}{2 \pi \alpha^{\prime} \omega} \operatorname{arcsine}(\omega r)
$$

or

$$
r=\frac{1}{\omega} \sin \left(\omega 2 \pi \alpha^{\prime} \sigma\right)
$$

The half length $R$ of the (open) string is of course the maximal achievable

$$
R=\frac{1}{\omega}
$$

Let us identify the usual internal string time $\tau$ with the target space time essentially and let us take the plane in which the string rotates to be the $X^{1} X^{2}$-plane. Then while the coordinates $X^{3}=X^{4}=\ldots=X^{25}=0$ we have

$$
\begin{aligned}
X^{1}(\sigma, \tau) & =\cos \left(\omega 2 \pi \alpha^{\prime} \tau\right) r \\
& =\frac{1}{\omega} \cos \left(\omega 2 \pi \alpha^{\prime} \tau\right) \sin \left(2 \pi \alpha^{\prime} \omega \sigma\right) \\
X^{2}(\sigma, \tau) & =\frac{\sin \left(\omega 2 \pi \alpha^{\prime} \tau\right) \sin \left(2 \pi \alpha^{\prime} \sigma \omega\right)}{\omega}
\end{aligned}
$$

To have a hope of having made a proper conformal gauge choice we take $\tau=\frac{t}{2 \pi \alpha^{\prime}}$.

If the solution ansatz which we have here made on physical ground is indeed a solution to the internal D'Alembertian equation

$$
\left(\frac{\partial^{2}}{\partial \tau^{2}}-\frac{\partial^{2}}{\partial \sigma^{2}}\right) X^{\mu}(\sigma, \tau)=0
$$

it shall be possible to resolve the $X^{\mu}$ into right and left mover like

$$
X^{\mu}(\sigma, \tau)=X_{R}^{\mu}(\tau-\sigma)+X_{L}^{\mu}(\tau+\sigma) .
$$

Indeed it is easy to use the formulas for taking sin and cos for sums of variables such as

$$
\sin \left(\omega \cdot 2 \pi \alpha^{\prime}(\tau \pm \sigma)\right)=\sin \left(2 \omega \pi \alpha^{\prime} \tau\right) \cos \left(2 \omega \pi \alpha^{\prime} \sigma\right) \pm \cos \left(2 \omega \pi \alpha^{\prime} \tau\right) \sin \left(2 \omega \pi \alpha^{\prime} \sigma\right)
$$

and

$$
\cos \left(\omega 2 \pi \alpha^{\prime}(\tau+\sigma)\right)=\cos \left(2 \omega \pi \alpha^{\prime} \tau\right) \cos \left(2 \omega \pi \alpha^{\prime} \sigma\right) \mp \sin \left(\omega 2 \alpha^{\prime} \pi \tau\right) \sin \left(\omega 2 \alpha^{\prime} \pi \sigma\right)
$$

to rewrite e.g.

$$
\begin{aligned}
X^{1}(\sigma, \tau) & =\frac{1}{\omega} \cos \left(\omega 2 \pi \alpha^{\prime} \tau\right) \sin \left(\omega 2 \pi \alpha^{\prime} \sigma\right) \\
& =\frac{1}{2 \omega}\left(\sin \left(\omega 2 \pi \alpha^{\prime}(\tau+\sigma)\right)-\sin \left(\omega 2 \pi \alpha^{\prime}(\tau-\sigma)\right)\right. \\
& =X_{L}^{1}(\tau+\sigma)+X_{R}^{1}(\tau-\sigma)
\end{aligned}
$$


where then

$$
X_{R}^{1}(\tau-\sigma)=\frac{-1}{2 \omega} \sin \left(\omega 2 \pi \alpha^{\prime}(\tau-\sigma)\right)
$$

and

$$
X_{L}^{1}(\tau+\sigma)=\frac{1}{2 \omega} \sin \left(\omega 2 \pi \alpha^{\prime}(\tau+\sigma)\right)
$$

Similarly we can write

$$
\begin{aligned}
X^{2}(\sigma, \tau) & =\frac{\sin \left(\omega 2 \pi \alpha^{\prime} \tau\right) \sin \left(\omega 2 \pi \alpha^{\prime} \sigma\right)}{\omega} \\
& =\frac{1}{2 \omega}\left(\sin \left(\omega 2 \pi \alpha^{\prime}(\tau+\sigma)\right)+\sin \left(\omega 2 \pi \alpha^{\prime}(\tau-\sigma)\right)\right. \\
& =X_{L}^{2}(\tau+\sigma)+X_{R}^{2}(\tau-\sigma)
\end{aligned}
$$

where

$$
\begin{aligned}
& X_{L}^{2}(\tau-\sigma)=\frac{1}{2 \omega} \sin \left(\omega 2 \pi \alpha^{\prime}(\tau-\sigma)\right) \\
& X_{R}^{2}(\tau+\sigma)=\frac{1}{2 \omega} \sin \left(\omega 2 \pi \alpha^{\prime}(\tau+\sigma)\right)
\end{aligned}
$$

At the ends of the string corresponding in the above notation to $\omega 2 \pi \alpha^{\prime} \sigma= \pm \frac{\pi}{2}$ we shall have that the tension in the string

$$
\text { "tension" } \propto X^{\prime \mu}(\sigma, \tau)=-\dot{X}_{R}(\tau-\sigma)+\dot{X}_{L}(\tau+\sigma)
$$

shall be zero, since there is nothing further out.

Calling

$$
\begin{aligned}
\tau_{R} & =\tau+\sigma \\
\tau_{L} & =\tau-\sigma
\end{aligned}
$$

We could write our above formulas

$$
\begin{aligned}
X_{R}^{1}\left(\tau_{R}\right) & =-\frac{1}{2 \omega} \sin \left(\omega 2 \pi \alpha^{\prime} \tau_{R}\right) \\
X_{L}^{1}\left(\tau_{L}\right) & =\frac{1}{2 \omega} \sin \left(\omega 2 \pi \alpha^{\prime} \tau_{L}\right) \\
X_{R}^{2}\left(\tau_{R}\right) & =\frac{1}{2 \omega} \sin \left(\omega 2 \pi \alpha^{\prime} \tau_{R}\right) \\
X_{L}^{2}\left(\tau_{L}\right) & =\frac{1}{2 \omega} \sin \left(\omega 2 \pi \alpha^{\prime} \tau_{L}\right)
\end{aligned}
$$

and boundary conditions of no tension becomes

$$
\dot{X}_{L}^{\mu}\left(\tau_{L}=\tau+\frac{\pi}{2 \cdot 2 \pi \alpha^{\prime} \omega}\right)=\dot{X}_{R}^{\mu}\left(\tau_{R}=\tau-\frac{\pi}{2 \cdot 2 \pi \alpha^{\prime} \omega}\right)
$$

the end of the string having $\sigma=-\frac{\pi}{2}$ obtain

$$
\dot{X}_{L}^{\mu}\left(\tau_{L}=\tau-\frac{\pi}{2 \omega 2 \pi \alpha^{\prime}}\right)=\dot{X}_{R}^{\mu}\left(\tau_{R}=\tau+\frac{\pi}{2 \omega 2 \pi \alpha^{\prime}}\right)
$$


For $\mu=1$ for example we see that these conditions are true because a shift in the $\tau$ argument $\tau_{R}$ or $\tau_{L}$ by 2 times $\frac{\pi}{2 \cdot \omega 2 \pi \alpha^{\prime}}$ corresponds to a shift by $\pi$ in the argument of the sine and that gives just the sign shift needed because $X_{R}^{1}\left(\tau_{R}\right)=-\frac{1}{2 \omega} \sin \left(\omega 2 \pi \alpha^{\prime} \tau_{R}\right)$ while $X_{L}^{1}\left(\tau_{L}\right)=\frac{1}{2 \omega} \sin \left(\omega 2 \pi \alpha^{\prime} \tau_{L}\right)$.

Apart from some shift in the argument the $X_{R}^{\mu}$ and $X_{R}^{\mu}$ are basically the same functional form - as we also see in our example — due to the boundary condition(s) at the end of the string.

Because we have at present a bit bad notation used $\sigma=\frac{\pi}{2 \cdot 2 \pi \alpha^{\prime} \omega}$ at one of the ends we get in fact

$$
\dot{X}_{L}^{\mu}\left(\tau_{L}\right)=\dot{X}_{R}^{\mu}\left(\tau_{R}=\tau_{L}+\frac{\pi}{2 \pi \alpha^{\prime} \omega}\right)
$$

If we had the end to have $\sigma=0$ we would have simply gotten

$$
\dot{X}_{L}^{\mu}\left(\tau_{L}\right)=\dot{X}_{R}^{\mu}\left(\tau_{R}=\tau_{L}\right) \quad(\text { for } \sigma=0 \text { at end }) .
$$

In any case we only need to use either $\dot{X}_{R}^{\mu}$ or $\dot{X}_{L}^{\mu}$ since they are of the same form.

\subsection{The closed string}

On the second translation on the figure we have taken as example a closed string in the configuration wherein it is put back and forth along the same piece of line, and it - really meaning its two pieces - rotate just like the open string just considered. Now the seeming end points are just accidental but not truly physically. In this case of closed we shall not identify the right and left movers. Rather each of them give rise to its own "cyclic chain". Therefore we have for this drawn two independent (although they happen to have the same coordinates) "cyclic chains". Let us stress the rule: to an open string corresponds only one cyclic chain, while to a closed string there corresponds two, one for the right mover modes and one for the left.

Concerning the above discussion it should be noted that we considered

$$
\begin{aligned}
& \dot{X}_{R}^{\mu}\left(\tau_{R}\right)=\frac{d X_{R}^{\mu}}{d \tau_{R}} \\
& \dot{X}_{L}^{\mu}\left(\tau_{L}\right)=\frac{d X_{L}^{\mu}}{d \tau_{L}}
\end{aligned}
$$

rather than $X_{R}^{\mu}\left(\tau_{R}\right)$ and $X_{L}^{\mu}\left(\tau_{L}\right)$ themselves - and that is something we for technical reasons, have decided to do in our Novel SFT - Had we not done that, the arguing away having both $X_{L}^{\mu}$ and $X_{R}^{\mu}$ in the open string would not have worked. So it were a quite important point to make this differentiation!

For illustration of our formulation/model for our novel string field theory you shall imagine drawing in 25 or 26 dimensional perspective the right mover field $X_{R}^{\mu}$ differentiated with respect to its variable $\tau-\sigma$, thinking classically.

To each open string right mover derivative is a 26 -vector being a periodic function with the period used for $\sigma$. The boundary condition at the end ensures that right and left mover derivatives are equal for the open string. 


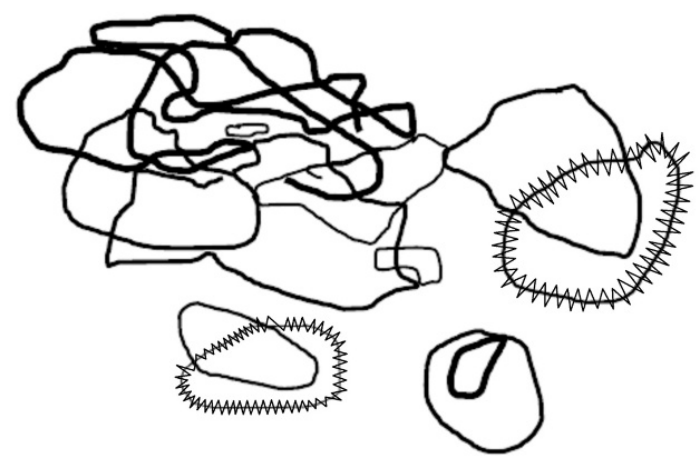

Figure 4. The images of $X_{R}$ and $X_{L}$ are represented by the closed curves called cyclic chains. The all curves do not have ends.

Thus we get to the open string a corresponding topologically circular figure the "cyclic chain". For a closed string one can both imagine drawing the right and the left mover and they become two in general different closed curve images (= two "cyclic chains").

For a single string in the "conformal gauge" we have the well-known equation of motion

$$
\left(\partial_{\tau}^{2}-\partial_{\sigma}^{2}\right) X^{\mu}(\sigma, \tau)=0
$$

and solve it by the splitting

$$
X^{\mu}(\sigma, \tau)=X_{R}^{\mu}(\tau-\sigma)+X_{L}^{\mu}(\tau+\sigma)=X_{R}^{\mu}\left(\tau_{R}\right)+X_{L}^{\mu}\left(\tau_{L}\right)
$$

As introduction to our Novel String Field Theory we shall imagine - and let us first think classically — that for each string development in time - in Minkowski space — draw a to such a moving/oscillating string corresponding image of the $\tau_{R}=\tau-\sigma$ and $\tau_{L}=\tau+\sigma$ derivatives $\dot{X}_{R}^{\mu}\left(\tau_{R}\right)$ and $\dot{X}_{L}^{\mu}\left(\tau_{L}\right)$ of these $X_{R}^{\mu}\left(\tau_{R}\right)$ and $X_{L}^{\mu}\left(\tau_{L}\right)$.

Because of the periodicity for finite size strings the two images of $X_{R}$ and $X_{L}$ will be closed curves, called "cyclic chains".

The meaning of the foregoing figure with the net of curves is, that there is in a perspectively drawn Minkowski space of $26=25+1$ dimensions (in bosonic string case). A point on the net each time some one of all strings present in Universe for some value of $\tau_{R}=\tau-\sigma$ or $\tau_{L}=\tau+\sigma$ the respective derivatives $\dot{X}_{R}^{\mu}\left(\tau_{R}\right)$ or $\dot{X}_{L}^{\mu}\left(\tau_{L}\right)$ of the string space time position field $X^{\mu}(\tau, \sigma)$ take their vectorial value equal to that point. This net of curves is thought classically at first: i.e. $\dot{X}_{R}^{\mu}\left(\tau_{R}\right)$ and $\dot{X}_{L}^{\mu}\left(\tau_{L}\right)$ have meaningful vectorial values once the last bit of gauge choice has been chosen. The reader is encouraged to first think of the net of curves ignoring quantum mechanisms, so that at least after a gauge choice the variables $\dot{X}_{R}^{\mu}$ and $\dot{X}_{L}^{\mu}$ have well defined values, that are $25+1$ vectors.

\subsection{One open string would contribute say the slim closed curve contained in the net}

For an open string one has at both end-points (say $\sigma=0,2 \pi$ )

$$
X^{\prime \mu}=-\dot{X}_{R}^{\mu}+\dot{X}_{L}^{\mu}=0
$$




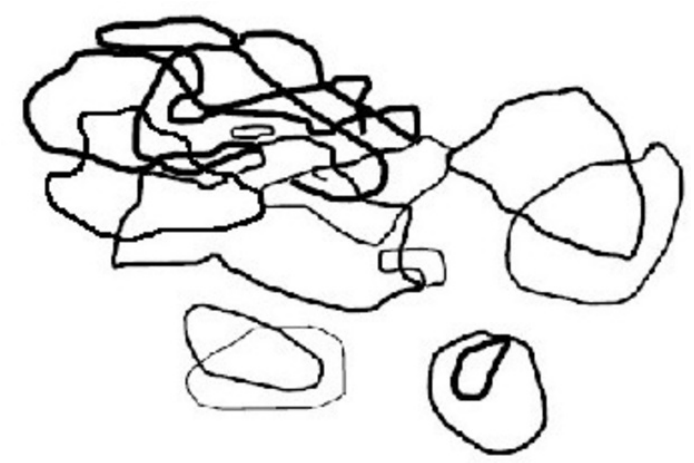

Figure 5. One open string depicted by the slim closed curve contributes in the net.

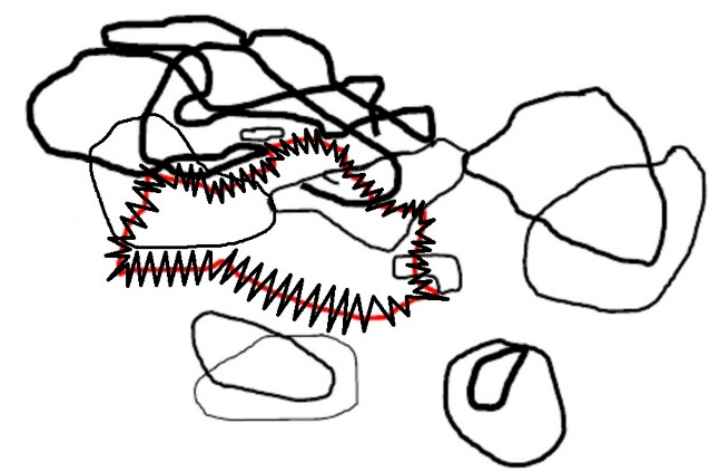

Figure 6. One more open string contribution is added as a wavy closed curve in the cyclic chain. All curves have no ends.

Since this must be true for all $\tau$, it is enough information to deduce that for the open string

$$
\dot{X}_{R}^{\mu}(\tau)=\dot{X}_{L}^{\mu}(\tau) \text {. }
$$

Thus at any moment of $\tau$ there is for each open string a closed circle, a "cyclic chain", of $\dot{X}_{R}^{\mu}$ or $\dot{X}_{L}^{\mu}$, so that we get for each open string a closed circle (the cyclic chain) of image points in the (perspectively imagined) $26=25+1$ dimensional space on the figure.

\subsection{We add one more — now wavy curve - open string contribution, a cyclic chain}

\subsection{Comments on our net of cyclic chains representing string via their right and left mover derivatives}

The whole set of all the right or left mover derivatives symbolized by the thin-curved network, can be interpreted as coming from various cyclic chains associated with various open string, or some pairs of them could correspond to closed strings.

We illustrated by various colors and thicknesses of the curves, how the image of all the right and left mover derivatives could be divided into contributions e.g. from different open strings. (some pairs of cyclic chains potentially correspond to just the same closed string instead of to two open ones).

We stress that the division into contributions from different cyclic chains, different open strings say, is not unique! 


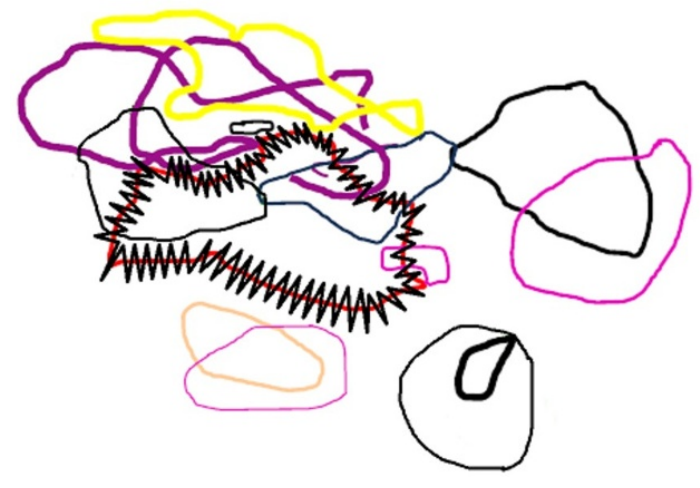

Figure 7. The whole set of all the right or left mover derivatives are symbolized by the thin-curved network. They can be interpreted as unification of a series of closed cycles that close each other.

\subsection{Further comments on novel string field theory model}

For seeing our model, our novel string field theory, it is the crucial point to imagine that the cyclic chains shown on our figures here in the $25+1=26$ dimensional space(-time) should be thought of as series of "objects" meaning a discretization of the cyclic chains into particle-like objects, that can be created or annihilated by creation and annihilation operators. So in this creation and annihilation we already now think quantum mechanically.

Thus the net of cyclic chains is truly thought of as represented by the effect of a lot of actions with creation or annihilation operators on a "background state" (=vacuum).

The spirit of our novel string field theory is most quickly presented by simply replacing the sites of $\dot{X}_{L}^{\mu}$ and $\dot{X}_{R}^{\mu}$ values representing a state of a universe with a number of open and closed strings called Im by a representation by a Hilbert space vector in a Hilbert space, in which what we call "objects" can be created and annihilated. The main point is the replacement

$$
\left.\operatorname{Im} \rightarrow \prod_{J \mu \in \operatorname{Im}} a^{+}\left(J^{\mu}\right) \mid \text { "vac" }\right\rangle
$$

written very shortly.

Here $J^{\mu}$ denotes a Minkowski space point and the product runs over those $J^{\mu}$ 's which lie in the union set Im.

There is, however, a series of technical troubles and the simple replacement

$$
\left.\operatorname{Im} \rightarrow \prod_{J \in \operatorname{Im}} a^{+}(J) \mid \text { "vac" }\right\rangle
$$

is to be considered an oversimplified pedagogical presentation, being quick correct.

First of all formulating a product of creation operators $a^{+}\left(J^{\mu}\right)$ requires that the product runs over a discrete set rather than a continuous set as $\mathrm{Im}$. So really we rather should say

$$
\left.\operatorname{Im} \rightarrow \prod_{I \in \text { discretized Im }} a^{+}\left(J^{\mu}(I)\right) \mid \text { "vac" }\right\rangle
$$

where we have split the "continuous" set Im into a large number of small pieces (or intervals in $\tau_{R}$ or $\tau_{L}$ 's) enumerated by essentially an integer $I$. 
Secondly what we would achieve then would not be a true quantum theory because $\dot{X}_{R}^{\mu}\left(\tau_{R}\right)$ say in quantum single string theory does not commute with itself for different $\tau_{R}$-values

$$
\left[\dot{X}_{R}^{\mu}\left(\tau_{R}\right), \dot{X}_{R}^{\nu}\left(\tau_{R}^{\prime}\right)\right]=i \delta^{\prime}\left(\tau_{R}-\tau_{R}^{\prime}\right)
$$

To be able to properly quantize both the single object system and second quantize, the discretized $J^{\mu}(I) \stackrel{\propto}{\sim} \dot{X}_{R}^{\mu}\left(\tau_{R}\right)$ should be so that they mutually commute. The trick to achieve such commutation since long proposed for our novel string field theory was to only use to give creation operators $a^{+}\left(J^{\mu}(I)\right)$ for the even I's. That is to say that the true expression becomes

$$
\left.\operatorname{Im} \rightarrow\left(\prod_{I \in \text { discretized Im and } I \text { even }} a^{+}\left(J^{\mu}(I)\right)\right) \mid \text { "vac" }\right\rangle
$$

Here the important point is that the $I$ variable only runs over every other of the at first twice as large number of bits/objects. Because the commutator $i \delta^{\prime}\left(T_{R}-T_{R}^{\prime}\right)$ after discretization gives lack of commutation between neighboring or objects we can achieve full commutation if we leave out every other of the bits, we call the left out ones the odd and denote them by odd integers.

A third minor technical problem is that we should like to have the objects be their own antiparticles (they should be "Majorana" so to speak) I.e.

$$
a^{+}\left(J^{\mu}\right)=a\left(-J^{\mu}\right)
$$

But if so we would with a "normal" quantization get

$$
\begin{aligned}
a^{+}\left(J^{\mu}\right)|" \operatorname{vac} "\rangle & =0 \\
\text { for } J^{0} & <0 .
\end{aligned}
$$

In fact it were the trouble of our calculation in which we only obtained one of the three terms in the Veneziano amplitude. To avoid that we should even be able add some negative energy to the vacuum | "vac" $\rangle$ i.e. we want

$$
\begin{aligned}
& a^{+}\left(J^{\mu}\right)|" \operatorname{vac} "\rangle \neq 0 \\
&\left(\text { even for } J^{0}<0\right)
\end{aligned}
$$

even when what we interpret $J^{0}$ the energy is negative.

It is this problem that shall be solved by using as vacuum a state with the property that $a^{+}\left(J^{\mu}\right)|0\rangle$ shoud not be zero. We call such a vacuum "the rough Dirac sea".

\subsection{The rough Dirac sea}

At first one would be tempted to think of the vacuum or "background state" for the objects as a state in which objects with positive energy (if that makes any sense) were the only ones possible to produce, but... : even though energy of a single object can be given a meaning, we shall assume that the "background state" for the second quantized objects-theory is 


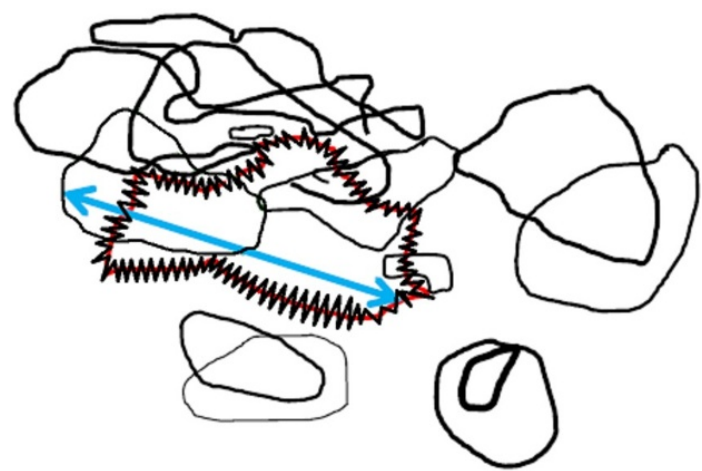

Figure 8. Imagining putting the double arrow to connect all pairs of one point on the blue and one on the red it would come through all the time track points on a closed string. For each pair run through the $\dot{X}_{R}^{\mu}$,s on the two rounds flux the tangent plane to the time track of the closed string.

not of the simple type that can only be modified to make the sum of the energies of the objects larger! Rather you can also add to it negative energy.

This is analogous to what we call the "rough Dirac sea"(see appendix).

On the last figure we show putting some arrows, how to construct the points or small pieces of the string time track - i.e. the surface in Minkowski space through which the string in question passes: to each pair of small bits on the cyclical chain corresponds a little area on the space - time track of the string.

The string time track is a two-dimensional manifold and thus one needs two onedimensional parameters to parametrize it. We use for the open string the same cyclic chain as being both parameters (two different point on the cyclic chain), while we for the closed string we use two different cyclic chains.

\subsection{Illustration of connection to the string}

We have put in on the picture figure 8 of the curve a narrow arrow. Such an arrow corresponds to a point on the string time track, or rather one point for each period of the string motion, in the sense that a couple of tangent vectors spanning the tangent to the string time track at the point in question are given by the two points in $25+1=26$ space(-time) at the two ends of the (double)arrow.

To obtain all the time track points of an open string modulo periodicity you must take all the arrows that can connect two points on the cyclical chain describing the open string in question.

To obtain those for a closed string you must use all the arrows connecting one point on one of the two cyclic chains to the other one.

On the figure illustrating the closed string in terms of two cyclical chains you obtain the tangent- basis vectors of the various infinitesimal pieces of the closed string time track by going through all possible arrows connecting one point on the cyclic chain, green, and one on the cyclic chain, red.

Again we get a two-dimensional time-track of a string -now closed- by having it parametrized by two parameters running along cyclic chains. 


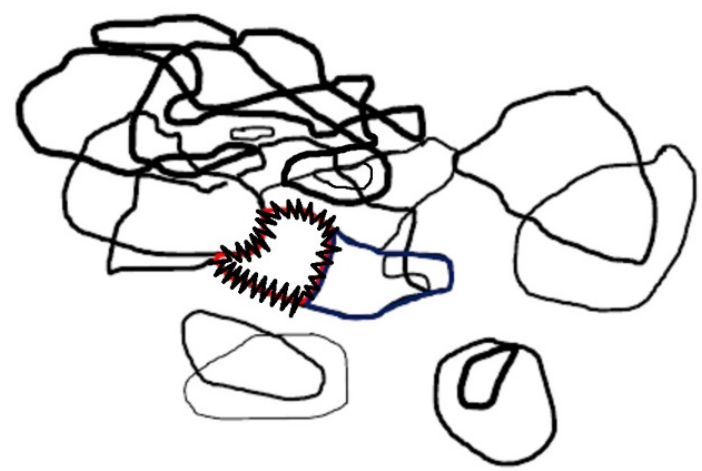

Figure 9. Imagine that we move around the blue double arrow in all possible positions connecting one point on the "zigzagged" loop and one point on the "thin" loop. It then runs through a twodimensional manifold of pairs of end-points, and to each such pair of end-points (of the double arrow) the associated $\dot{X}_{R}{ }^{\mu}$-pair specifies a twodimensional plane spanned by the pair of $\dot{X}_{R}{ }^{\mu}$ 's. The set(manifold) of all these planes makes up the set of tangent planes to the time-track (imbedded in the $25+1$ dimensional physical Minkowsky space-time) for the closed string development in question.

What happens if we first by creation or annihilation operator insert a piece of cyclic chain with one momentum distribution and then add the one with just the opposite one? Actually they cancel and it becomes as if nothing had been done.

This opens up a strange possibility for inserting the cyclic chains corresponding to a couple of say open strings: we could let a piece of the cyclic chain corresponding to one of the two strings happen to be just the "opposite" of a piece of the cyclic chain for the other open string.

In that case inserting the cyclic chains corresponding to the two open strings leads to there being two pieces of cyclic chains canceling each other.

And thus the final state in our $25+1=26$ dimensional space for "objects" would have got zero objects along the piece of cancellation. And the latter would not be marked in our Hilbert space for second quantized object.

One would only there "see" the pieces that were not canceled.

Having in mind the possibility of canceling two "opposite pieces of cyclic chain we can without any trace in our second quantized object-state have two (oppositely oriented but otherwise locally in the same state) pieces of the blue and the red cyclic chains present.

On the above figure these canceling pieces are illustrated with the curve where the blue and the red curves follow each other.

That there along this piece is no sign of the cyclic chains in the object-description is illustrated by there being no thin black curve along this piece before we drew in the red and the blue circles (cyclic chains) anywhere on top of the thin black curves illustrating the second quantized object-state.

\section{The state of several strings}

At first — and that we did in our earlier work — in a Bled proceedings [41] —- one would simply take the second quantized Hilbert space state as the incomming two particle state 
$\mid \vec{p}_{1}, \vec{p}_{2}$, inc $\rangle$ to be the one obtained from the object formulation vacuum $|0\rangle$ by first acting with the creation operator for the cyclically ordered chain.

$$
\begin{aligned}
C^{+}(\text {string } 1)= & \int_{\begin{array}{c}
\text { around cyclic } \\
\text { chain } I_{e} \text { even }
\end{array}} \Psi\left(J^{\mu}(0), J^{\mu}(2), \ldots, J^{\mu}(N-2)\right) \\
& \cdot a^{+}\left(J^{\mu}(0)\right) a^{+}\left(J^{\mu}(2)\right) \cdots a^{+}\left(J^{\mu}(N-2)\right) \prod_{I_{e}^{\text {even }}} d J\left(I_{e}\right)
\end{aligned}
$$

on this vacuum $|0\rangle$ and then successively act with the analogous creation operator for string 2 , say $C$ (string 2 ). I.e. the description in our picture should be

$$
C^{+}(\text {string } 2) C^{+}(\text {string } 1)|0\rangle
$$

\subsection{A formalism of replacement of ghost}

If we imagine working with formulation with ghosts the $J^{\mu}\left(I_{e}\right)$ 's must be replaced by constructions such as $\left(J^{\mu}\left(I_{e}\right), \uparrow, \downarrow\right)$ or $\left(J^{\mu}\left(I_{e}\right), \downarrow, \downarrow\right)$ also involving ghost (but that is not so important just now) .

If one works with our old infinite momentum frame it would only be the transverse components and you would write instead

$$
C^{+}(\operatorname{string} 1)=\int \Psi\left(\vec{J}_{T}(0), \vec{J}_{T}(2), \ldots, \vec{J}_{T}(N-2)\right) \prod_{\substack{I_{e} \text { even } \\ \text { around circle }}}\left(a^{+} \vec{J}\left(I_{e}\right)\right)\left(d \vec{J}_{T}\left(I_{e}\right)\right)
$$

If our creation operators for the objects like $a^{+}\left(\vec{J}_{T}\left(I_{e}\right)\right)$ all add say a positive energy rather than like the ones in say the BRST formalism $a^{+}\left(J^{\mu}\left(I_{e}\right)\right.$, ghost), then there is no way that the products of a couple of creation operators for objects could be simplified. If we however have operators like $a^{+}\left(J^{\mu}\left(I_{e}\right), \uparrow, \downarrow\right)$ that are a priori able to bring energy and momentum of any sign, e.g. also negative energy $J^{0}$, then there is opened the possibility that the action of a couple of them

$$
a^{+}\left(J^{\mu}\left(I_{e}\right), \cdot, \cdot\right) a^{+}\left(-J^{\mu}\left(I_{e}\right), \cdot, \cdot\right) \propto 1
$$

could act proportional to a c-number!

This is the crucial progress by not fixing energy nor longitudinal momenta to be positive. For this to work it is crucial that two opposite 26-J creation operators both are nontrivial.

I.e. both say

$$
a^{+}\left(J^{\mu}, \cdot, \cdot\right)|0\rangle \neq 0
$$

and

$$
a^{+}\left(-J^{\mu}, \cdot, \cdot\right)|0\rangle \neq 0
$$

That requires a "rough Dirac sea". But provided - as we think we have - we have provided such nontrivial but opposite operator a "Majorana" boson theory for the objects say then one of the two operators $a^{+}\left(J^{\mu}, \cdot, \cdot\right)$ and $a^{+}\left(-J^{\mu}, \cdot, \cdot\right)$ may be considered the annihilation operator for the particle / here object created by the other one. Thus indeed we may argue

$$
a^{+}\left(-J^{\mu}, \cdot, \cdot\right) a^{+}\left(J^{\mu}, \cdot, \cdot\right)|0\rangle=|0\rangle
$$




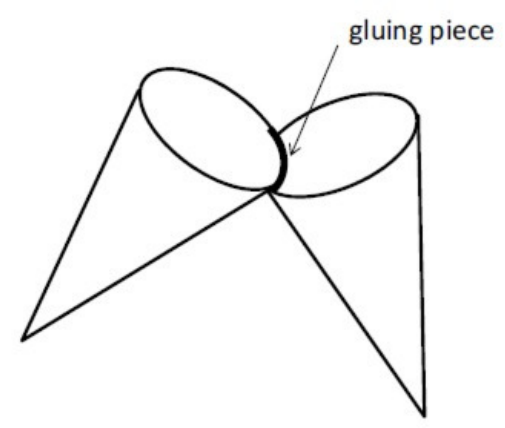

Figure 10. The two cones can be glued together at the common edges.

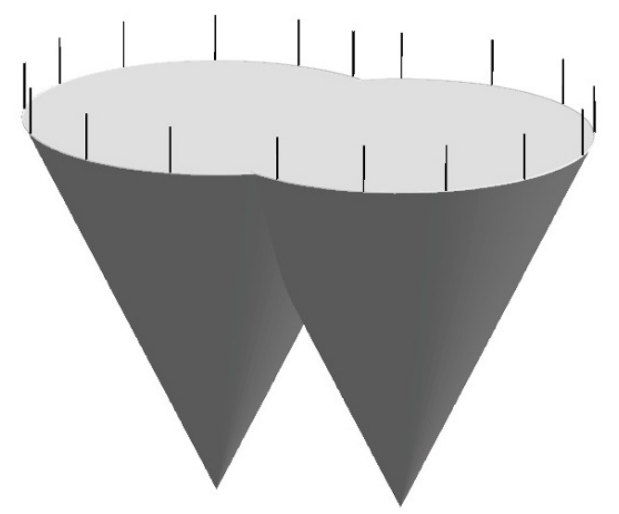

Figure 11. The two cones can have a common edge as depicted.

so that the product acts as the unit operator. With continuum normalization one may have $\delta$-functions, but let us postpone this issue.

This means that in our "rough Dirac sea" picture we have to count that "oppoiste" (meaning opposite $J^{\mu}$, i.e. $J^{\mu}$ and $-J^{\mu}$ creation operators multiplied with each other can be replace - by calculation — by just unit operator.

\subsection{A trick of calculating the wave function}

Now we should remember that our crucial trick to calculate the wave functions $\Psi_{1}$, and $\Psi_{2}$ for our strings 1and 2 was to express them by means of imaginary $\tau$ functional integrals - so as to let only the ground state of the strings survive.

If a couple of strings have it so that their associated creation operator cyclic chains can partly annihilate in the sense of giving unit operators as just described, then rather than being left in the two-string-describing state these objects can be removed provided they are "opposite".

The calculation of the amplitude for what the removing object creation operators can be well now by a functional integral for the complex (=imaginary $\tau$ ) developments (of the cyclically ordered chains) but with the extra rule: piece(s) of the edge for string 1 half cylinder could be glued together with piece(s) of the edge for the half cylinder for string 2 half cilinder. 


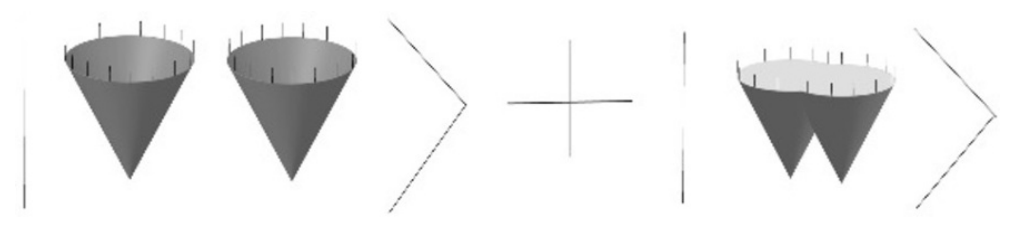

Figure 12. Two string states are sum of two state vectors as expressed in the figure.

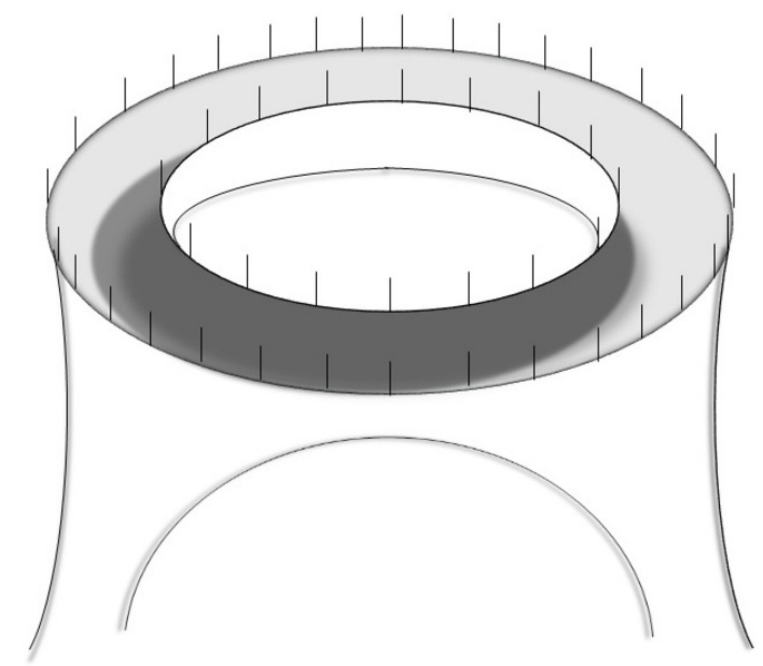

Figure 13. Topologically different one obtained by making use of two cones in the manner described in main text.

We would then - being closer to the classical solution — rather think of the figure as describing the functional integral to derive the resulting true object description for two string state

$$
\left.\mid \text { str. } 1 \text {, str. } 2, \underset{\text { com }}{\text { com }}\rangle=C^{+}(\text {string } 2) C^{+} \text {(string } 1\right)|0\rangle
$$

The possibility that there were nothing annihilated should not be neglected, since it is not necessarily negligible. So rather symbolically we can write these two terms as on figure 12 .

These more complicated terms are obtained by taking from each of two incoming strings; string 1 and string 2 more than one piece of their cyclic chains and then we use the rule

$$
a^{+}\left(J^{\mu}, \cdot, \cdot\right) a^{+}\left(-J^{\mu}, \cdot, \cdot\right) \sim 1
$$

along these - more than one - pieces. The typical construction such a more complicated term - namely corresponding to two pieces along which the rule (2.9) is used. It would lead to a contribution to the Hilbert space for the second quantized even object given by a functional integral for $J$ 's (or П's) being fields on a two dimensional Euclidean manifold.

This drawing is meant to be obtainable topologically from the one for piece on which (2.9) were used only by taking a two dimensional piece of the "bottom" pull it up while it hangs together and then chop off the cap of the pull out.

The two cyclic chainshas now to be identified with corresponding ones in the final state if we shall use our $\mid$ string 1 , string 2 ,inc.) to make an $S$-matrix element. 


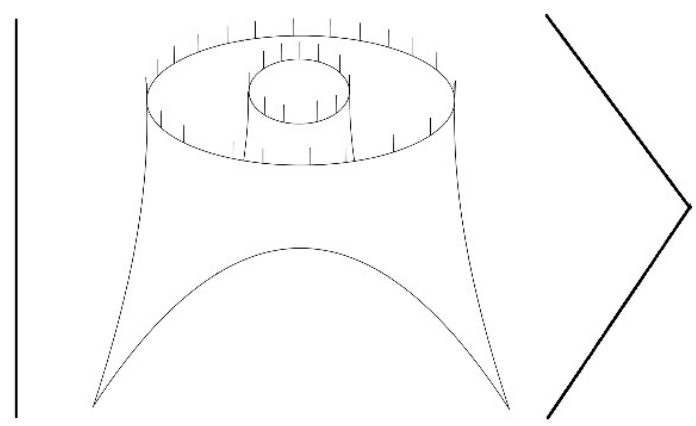

Figure 14. The Hilbert vector corresponding figure 13, to be included when calculating S-matrix element.

\section{An encouraging step; the Veneziano model except for the integration weight}

Having argued for functional-integral constructions as suggested by the above and with imaginary time, we have really obtained, what is usually considered the correct description of string development in a double description. The functional integrals are in our present philosophy just a mathematical trick to describe the wave function for the string states, while if you consider the functional integrals the usual way the surfaces drawn rather represent the development of the strings, even if in some way Wick rotated though. One shall note that in our philosophy there is in our object formulation no development. It is only a trick to construct the wave function. Nevertheless, there is an obvious similarity between the diagrams describing functional integrals which we obtain when we write down or describe the overlap between an initial $|i\rangle$ and a final $|f\rangle$ states in terms of the functional integrals and to the same $S$-matrix element corresponding development diagrams.

From this actually quite perfect correspondence we can in fact as a beginning derive that we must obtain the Veneziano [51] model(s) in question up to an extra factor under the integration sign. In the integral expression for the Veneziano model is more delicate to obtain. We believe that this inclusion of such pieces of cyclic chains cancelling each other so that no track of their existence is left in our description by means of objects can be of help to solve a problem, which we met in our development of our string field theory based on such object description.

The problem were the following: we sought to calculate from our novel string field theory the scattering of two particles into two others expecting to obtain a Veneziano model with three terms corresponding to the usual three pairs among the channeles $\mathrm{s}$, $\mathrm{t}$, and $u$. But $\cdots$ We got only one of these terms!

We did the calculation, that turned out in this way unsuccessfully in an infinite momentum frame "gauge" (=parametrization) choosing the right mover and the left mover coordinates ensuring a fixed amount of the "longitudinal" component of the 26-momentum for all objects.

Thus there were in this "gauge" choice no way to have the "longitudinal" component of momentum made opposite. 
So there were no way in that "gauge" to realize the "Phantasy or cancellation pieces of cyclic chain".

All cyclic chains corresponding to (open) strings would have to be "visible" in the second quantized object description.

But then two strings cannot become one by partial annihilation in the cyclic chain description.

In principle our "Novel String Field Theory" should just be a rewriting of a system of many strings interacting with each other. There should be nothing logically new, only reformulation! Whether we really have logical perfect correspondence (after quantization) depends, however, on how much information we count it that there is in our formalism. Strictly speaking we could make "philosophically different versions" of our model, each including different amounts of information in them. Only the one with large amount of information would match usual string theory. But we suggest to take the version with minimal amount of information most serious as our novel string field theory.

\section{The classical approximation summary of "layers of existence degrees"}

But in our formulation, the "Novel String field Theory" it is we think pedagogical to consider several layers of truth or existence corresponding to different versions with respect to including information into the formalism. Let us first describe these existence - "layers" in our classical (by classical formulation we have in mind that the single particle states are described classically with both $\Pi$ and $J$ having values simultaneously — in disagreement with Heisenberg uncertainty principle - while the objects are still second-quantized, so that a state in superposition of having different numbers of objects is in the picture) formalism, with which we started.

We have a series of steps from truly existing in our novel theory to being more and more phantasy, not really existing:

1. Fully existing the system of objects that can have both negative and positive energy - because they sit on a background of the "rough Dirac sea" (which is also fully existent, although we avoid having to go in detail formulating it.)

2. Chaining of Objects into Cyclical chains from the continuity of the strings and the boundary conditions we have the objects forming cyclically ordered chains with objects sitting with neighbor distances of the order of the "latticification cut off distance". Really we do not take it in our model that this chaining order has any physical existence in itself; but seeing a pattern of the "truly" existing objects with their $J^{\mu}$ 's and $\Pi^{\mu}$ 's we may let the nearness define for us a chaining. (A wrong way of chaining may lead to bad continuity).

3. Pairing by this "pairing" we mean the information telling, which cyclically ordered chains together corresponds to a string. Open strings come from just one cyclically ordered chain each, while closed strings each need two, but here the total 26-momenta for the two shall be the same. So again knowing the cyclic chains there is some basis/restriction for guessing, which ones to combine. 


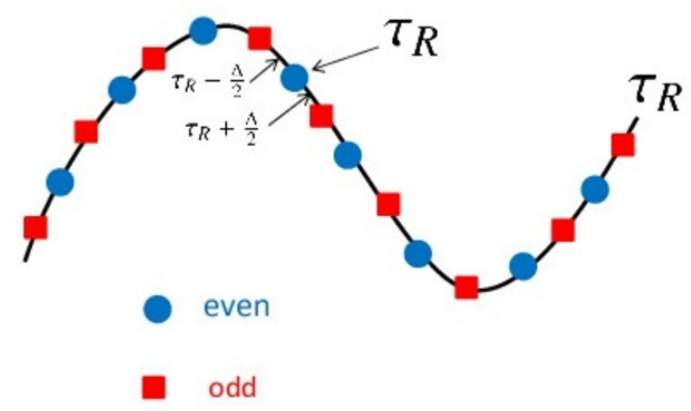

Figure 15. The string consists of a series of even and odd objects along the string alternatively put. As is depicted as an example the string is parametrized in the following manner. The right mover is parametrized by $\tau_{R}$. If the one even object is in $\tau_{R}$, the neighboring two objects are odd ones: one of them is situated at $\tau_{R}-\frac{\Delta}{2}$ and the other is at $\tau_{R}+\frac{\Delta}{2}$, where $\Delta$ is the cut off length into objects.

As told: we first attempted a description with the single object - and also single string - being treated classically, but allowing quantum mechanics in the second quantization, so that we could make a superposition of even different numbers of objects or strings.

While in Thorn's bits from pieces of sigma have positions commuting with each other, the right-mover part of the position does not commute with itself. Rather there is for its derivative - which we want to work with a delta-prime function commutator.

If we shall have seperate creation and annihilation operators objects in any state, it would at least be a very unwanted complication if the degrees of freedom for one object and another one did not commute. Thinking classically on the single object state we should thus have zero Poisson bracket between the variables associated with two different objects. This is, however, impossible if we want the objects to represent at least the $\tau$-derivative of say the right mover part of the string position field $\dot{X}_{R}^{\mu}\left(\tau_{R}\right)$, because these rightmover fields or their derivatives for different values of the argument $\tau_{R}\left(\tau_{R}\right.$ is usually replaced by a complex variable $\bar{z}$ ) do not commute, thus do not have zero Poisson bracket.

Wanting

$$
J_{R}^{\mu}\left(\tau_{R}\right) \propto \dot{X}_{R}^{\mu}
$$

we define

$$
J_{R}^{\mu}\left(\text { for interval bit }\left[\tau_{R}-\Delta / 2, \tau_{R}+\Delta / 2\right]\right)=X_{R}\left(\tau_{R}-\Delta / 2\right)-X_{R}\left(\tau_{R}+\Delta / 2\right),
$$

where $\Delta$ is our cut off "length" in "bits" or "objects".

But these $\dot{X}_{R}^{\mu}$, s do not commute, but rather

$$
\left[\dot{X}_{R}^{\mu}\left(\tau_{R}\right), \dot{X}_{R}^{\mu}\left(\tau_{R}^{\prime}\right)\right]=i \delta^{\prime}\left(\tau_{R}^{\prime}-\tau\right) \neq 0
$$

Replacing the $\tau_{R}$ parametrization of right movers - replacing $\bar{z}$ which were complex - by a discrete counting $I$ taking integer values our discretized object variables

$$
J_{R}^{\mu}(I)=X_{R}^{\mu}\left(\tau_{R}(I)+\Delta / 2\right)-X_{R}^{\mu}\left(\tau_{R}(I)-\Delta / 2\right),
$$


where say $\tau_{R}(I)=$ constant $+I * a$, obey crudely at least that even $I$ object variables $J_{R}^{\mu}(I)$ commute with each other, and the odd ones commute with themselves. However the even ones do not commute with their two odd neighbors! This is just describing a discretized delta-prime function.

We dueled in constricting our SFT to only consider the even numbered objects as independent objects. Then we let the variables - especially $J_{R}$ for the odd objects be written in terms of the conjugate variables $\Pi^{\mu}(I)$ of the neighboring even object variables:

$$
J_{R}^{\mu}(I)(\text { for odd } I)=-\alpha^{\prime} \pi\left(\Pi^{\mu}(I+1)-\Pi^{\mu}(I-1)\right) .
$$

the $\Pi(I \pm 1)$ to be used here are numbered by the even numbers $I \pm 1$ and thus can be the conjugate momenta of the assigned $J_{R}^{\mu}(I \pm 1)$ to the even objects respectively.

We can consider this expression for the odd $J_{R}$ as a kind of "integrating up" the odd $J_{R}$ to construct/give us the ח's. For an even value of the integer $K$ we solve our prosed equation for the odd $J_{R}$ 's

$$
\Pi_{R}^{\mu}(K)=\frac{J_{R}^{\mu}(K-1)+J_{R}^{\mu}(K-3)+J_{R}^{\mu}(K-5)+\cdots}{-\alpha^{\prime} \pi} .
$$

It looks that with this "integrating up" information on the original continuum string variable $\dot{X}_{R}^{\mu}\left(\tau_{R}\right) \sim J_{R}^{\mu}$ has been moved away in a non-local way for odd discrete points and is stored as the $\Pi_{R}^{\mu}$ for even argument.

It may be less serious though since $\dot{X}_{R}^{\mu}\left(\tau_{R}\right) \sim J_{R}^{\mu}$ were already differentiated, so really the $\Pi_{R}^{\mu}$ becomes essentially the right mover part of the position variable for the string.

If our so called string field theory is only a theory of essentially free massless objects, then it is a mystery: where is the string?

Do we even get the Veneziano model out of it? Yes we do. We actually can calculate to obtain Veneziano model - actually though for an overlap between initial and final state rather than for a complicated S-matrix.

\section{Main idea in calculating Veneziano amplitude}

Since nothing goes on the S-matrix can only be unity and the S-matrix element just an overlap of in and outgoing states $\langle f \mid i\rangle$.

We write these in or outgoing states by having for each particle a wave function in terms of objects.

These wave functions are written by means of an imaginary time functional integral for a string extracting the ground state (of the string) by it surviving long imaginary time development.

We wrote of the overlap becoming the scattering aplitude as the various pieces of surfaces with corresponding functional integrals, that were used to deliver the wave functions in object formulation corresponding to the incomming string, and to the outgoing ones. By looking at the various cases we see that for the

$$
1+2 \rightarrow 3+4
$$




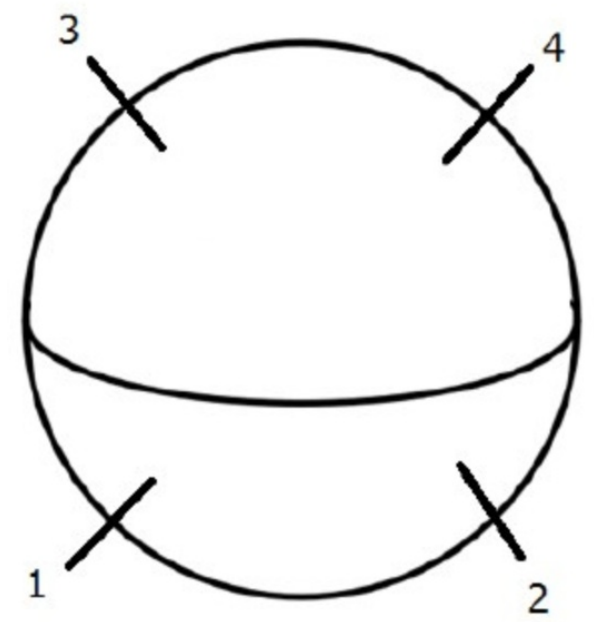

Figure 16. Illustration of four ground state scattering $1+2 \rightarrow 3+4$ where the surface is an $S^{2}$-sphere.

scattering of ground-state (tachyons, in bosonic string theory), we realize at first that the combined surfaces modulo conformal and topology conserving transformations consists of an $S^{2}$-sphere with the four external ground state particles/string being attached to this $S^{2}$-sphere.

Because of the conformal invariance - up to an anomaly - of the funcitonal integral the possible inequivalent configurations of the four external lines on the Riemann-sphere $S^{2}$ is given by a single complex anharmonic ratio

$$
A=\frac{z_{1}-z_{3}}{z_{2}-z_{3}}: \frac{z_{1}-z_{4}}{z_{2}-z_{4}}
$$

where the $z_{i}$ 's are the Riemann surface notation places for the four external lines. By considering the construction in more detail one can see that in fact this anharmonic ratio $A$ becomes real and that all possible real values of $A$ can become realized. By the three values

$$
A=0,1, \infty
$$

corresponding to that a couple of external line attachments coincide the real axis compactified to the topology of an $S$ circle is divided into three parts.

Each of these three pieces would, provided we obtain the right external-momentumindependent-measure $\rho$ say, give rise to a term in the scattering amplitude as there are in the Veneziano model

$$
g^{2}(B(-a(t),-\alpha(s))+B(-\alpha(s),-\alpha(u))+B(-\alpha(u),-\alpha(t))) .
$$

For instance with an appropriate definition of an anharmonic ratio of the type of $A$, namely

$$
X_{\mu t}=\frac{z_{1}-z_{4}}{z_{1}-z_{2}}: \frac{z_{3}-z_{4}}{z_{3}-z_{2}}
$$




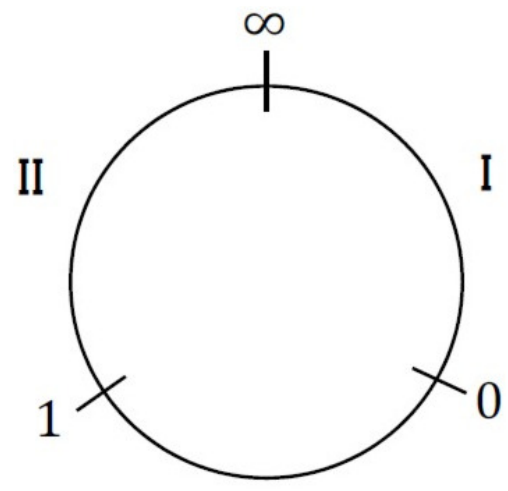

III

Figure 17. Inequivalent configurations of the four external lines on the Rieman-Sphere $S^{2}$ are $A=0,1, \infty$. The external line attachments is divided. $S^{2}$ topology's circle is divided into three intervals: I, II and III.

where we use the enumeration of external lines for $1+2 \rightarrow 3+4$ and call the $14-$ or $23-$ channel the $t$-channel

$$
\left(-p_{1}+p_{4}\right)^{2}=\left(-p_{2}+p_{3}\right)^{2}=t
$$

and the 13 - or 24 -channel the $u$-channel

$$
\left(-p_{1}+p_{3}\right)^{2}=\left(-p_{2}+p_{2}\right)^{2}=u
$$

we obtained in our previous article [44] that we got such an extra measure $\rho$ that the amplitude piece coming from

$$
0 \leq X_{\mu t} \leq 1
$$

because

$$
\int_{0}^{1} X_{\mu t}^{-\alpha_{t}(t)^{-1}}\left(1-X_{\mu}\right)^{-X_{u}(u)^{-1}} \rho\left(X_{\mu t}\right) d \alpha_{\mu t}=B(-\alpha(t),-\alpha(u)) .
$$

Our hope is that, an evaluation of the external momentum independent factor $\rho$ for the two other regions in the anharmonic ratio, will turn out to deliver the two missing terms, $B(-\alpha(s),-\alpha(t))$ and $B(-\alpha(s),-\alpha(u))$.

We should stress that we have already by referring to usual string scattering theory that the factor in the integrand depending on the external momenta has the correct form for giving these missing terms. This is already extremely promissing for that we shall obtain the full Veneziano model.

Let us also stress that in fact the two missing terms come from those terms in the overlap which in the object representation have parts of cyclic chains corresponding to different, say incoming strings just compensating/annihilating each other. So the two missing terms in our previous paper only come about, because we now have - due to rough Dirac sea - (See appendix A and B) the possibility of cyclic chain pieces with also negative energy. 


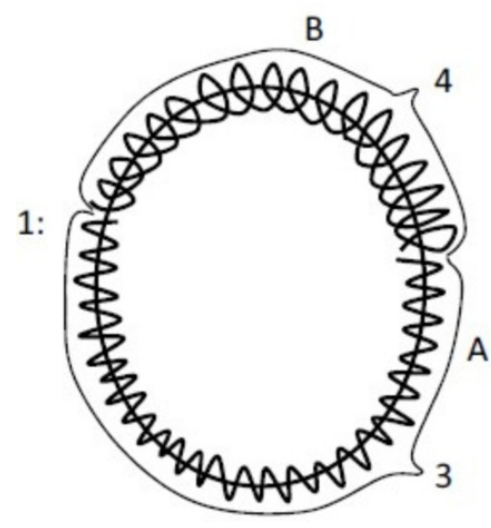

Figure 18. All the objects of the cyclic chain of string 1 must go to either 3 or 4 and the separation objects on the cyclic chain of string 1 fall into two pieces 3 or 4 .

\subsection{The simplest case of only positive $J^{+}=\frac{a \alpha^{\prime}}{2}$ (even) objects}

here as a starting example of calculating Veneziano model we consider the case that two incoming open strings have only postive $J^{+}=\frac{a \alpha^{\prime}}{2}$ objects in their cyclic chains, and also the two outgoing strings 3 and 4 have only positive $J^{+}$objects.

Then all the objects of the cyclic chain of string 1 must go to either 3 or 4 and the separation with fewest breakpoints (points where one class of objects stop and a new class begins) means that the objects on the cyclic chain of string 1 just falls into two pieces. One of these two pieces go into the final state string 3 , the other one goes into string 4 .

By saying the same for string 2 you soon end up with scheme figure 19.

How many objects there are in the four different classes marked by their curve signatures , and , are denoted by $N_{1}, N_{\infty}, N_{\infty 0000}, N_{-}$ respectively and we have assuming the easily derivable rule that the 26-momentum of the open string

$$
P_{\text {string }}^{\mu}=\frac{1}{2 \pi \alpha^{\prime}} \sum_{I=0}^{N-1} J^{\mu}(I)
$$

that the "longitudinal momenta" of the four strings (We use a light cone or metric notation with $\eta^{+}=\eta^{0}+\eta^{25}$ and $\eta^{-}=\eta^{0}-\eta^{25}$ )

$$
\begin{aligned}
& P_{\text {string 1 }}^{+}=\frac{a \alpha^{\prime}}{2 \Pi \alpha^{\prime} \cdot 2}\left(N+N_{\text {and }}\right)=\frac{a}{4}\left(N+N_{\text {and }}\right) \\
& P_{\text {string } 2}^{+}=\frac{a}{4}\left(N_{\ominus 000 \ominus}+N \_\right) \\
& (*) P_{\text {string } 3}^{+}=\frac{a}{4}\left(N_{\ominus 0000}+N_{\text {AnAMA }}\right) \\
& P_{\text {string } 4}^{+}=\frac{a}{4}\left(N \_+N_{1}\right)
\end{aligned}
$$

Having in mind that we shall calculate the Veneziano amplitude for one set of external 26momenta at a time, we must get the amplitude for one such fixed set of external momenta with a sum running over of the various possible combinations $\left(N_{1}, N_{\infty}, N_{\ominus 000 \ominus}\right.$, 


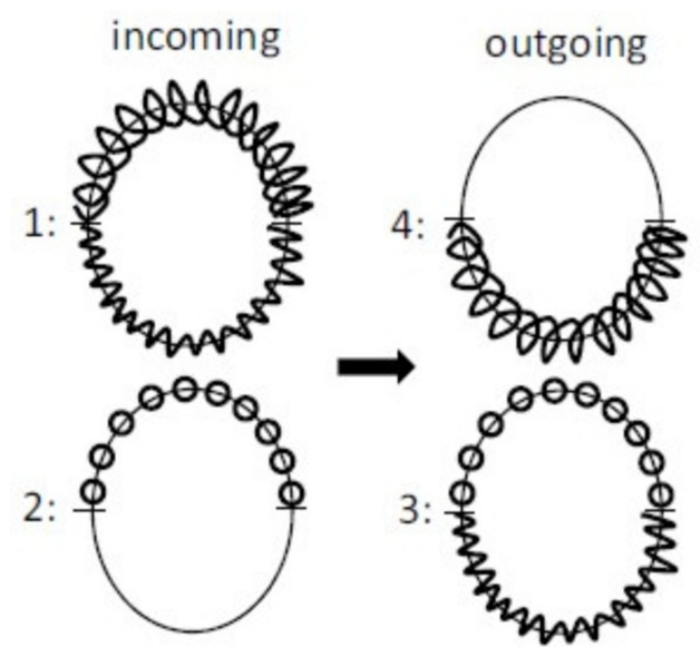

Figure 19. These figures illustrates how we think of the four cyclically ordered chains corresponding to the two incoming, 1 and 2, and the two outgoing, 3 and 4, strings in the scattering process, are divided into pieces according to where they are going or coming from. That is to say we denoted by one signature that series of objects on the cyclically ordered chain corresponding to string 1 which go into the cyclically ordered chain corresponding to the outgoing string 4, say. Similarly the other pieces going to or coming from a specific cyclically ordered chain and going to a specific other one have got a distinguishable signature. The arrow symbolizes the transition from initial to final states.

$N \_$) obeying these equations (*) for the fixed external momenta. This means obviously that the variations under the summation obey

$$
\Delta N=-\Delta N_{\text {anA }}=+\Delta N_{\ominus 0000}=-\Delta N
$$

Thinking of the $N_{1}, N_{\text {nan }}$, etc. as true numbers of objects, they cannot become negative. One should have in mind that there is only one free variable say $N_{\text {to }}$ sum over. To achieve the most simple range over which to sum we would much like that $N_{\text {and }}$ and $N$

would also go to zero simultaneously, in the opposite end of the integration/summation so to speak. But from $\left(^{*}\right)$ this wish would imply $P_{1}^{+}=P_{3}^{+}$and $P_{2}^{+}=P_{4}^{+}\left(\right.$from $N_{1}=$ $N_{\ominus 000 \ominus}=0$ simultaneously), while $N_{\text {anar }}=N_{\perp}=0$ simultaneous imply $P_{1}^{+}=P_{4}^{+}$ and $P_{2}^{+}=P_{3}^{+}$. All together these wish-requirements thus mean

$$
P_{1}^{+}=P_{2}^{+}=P_{3}^{+}=P_{4}^{+}
$$

If we trusted that the theory of ours were Lorentz invariant we might achieve this equality (5.13) by an appropriate Lorentz transformation.

If we do not have this case of equal $P^{+}$'s there can be different ways of connecting the objects from initial to final state and it may get more complicated.

Then under the assumption of the "longitudinal momenta" $P_{\kappa}^{+}$for $\kappa=1,2,3,4$ being all equal we obtain just a summation over say $N_{1}$ running over all integers from 0 to $4 a^{-1} P_{1}^{+}=4 a^{-1} P_{2}^{+}=\ldots=4 a^{-1} P_{4}^{+}$. 


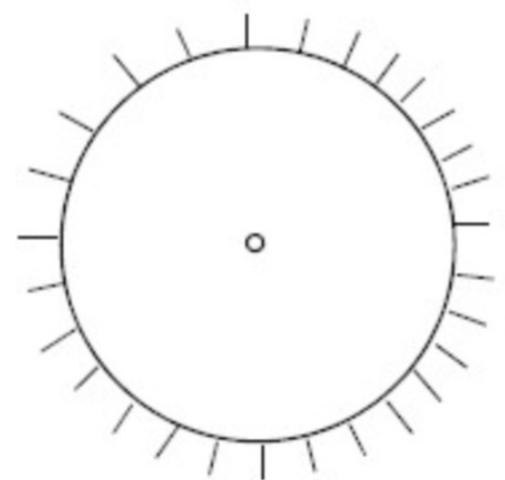

(a) A unit disk with the center punctured out

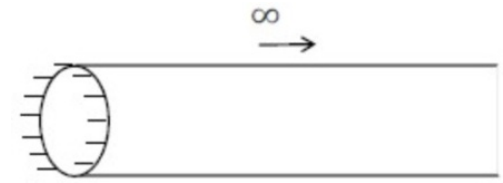

(b) A half infinite cylinder

Figure 20. (a) and (b) are conformally equivalent.

We shall approximate this summation by an integration

$$
\sum_{I \in\left[0,4 a^{-1} P_{1}^{+}\right]} \rightarrow \int d N
$$

and from here obtain the integration so characteristic for the Euler's Beta function form of cntributions to the Veneziano model.

A lot of details of the set up of the calculational procedure is to be found in [8-10]. The main point is that one first write the wave function for the set of even objects $N_{\text {a s }}+N_{\text {string }} / a$ of them, or rather only $2 P_{\text {string }} / a$ of them when we only want the even ones - by being given by the value of a functional integral being a regularized (cut off e.g. by a lattice) Feynman-Dirac-Wentzel one with the Nambu action or better one already made into a conformal gauge form

$$
\int \exp \left(-\left(2 \pi \alpha^{\prime}\right)^{-1} \int_{A}\left(\vec{\partial} \phi^{\mu}\left(\sigma^{1}, \sigma^{2}\right)\right) d \sigma^{1} d \sigma^{2}\right) D \phi^{\mu}
$$

(The index $\mu$ is the Lorentz index; but basically one develop the different factor with different $\mu$ separately; at the end multiply then.)

$A$ is chosen to be either a (unit) disk with the center punctured out or what is via conformal transformations a half infinite cylinder.

By this conformal transformation the punctured center corresponds to the cylinder running to infinity end.

Along the edge of the disk or conformally equivalent the finite end of the half infinite cylinder the objects in the cyclic chain corresponding to one of the strings, say string 1 , are imagined attached in a equidistant.

The meaning of this attachment to the edge is that the values $J^{\mu}(I)$ associated with objects around the cyclic chain are identified with differencec of neighboring values of the functional integral (dummy) variable $\phi^{\mu}$ (see equation (5.15). If there are in total $N$ objects in the cyclic chain the attached object $I$ may be at the angle-along the disk-edge- 


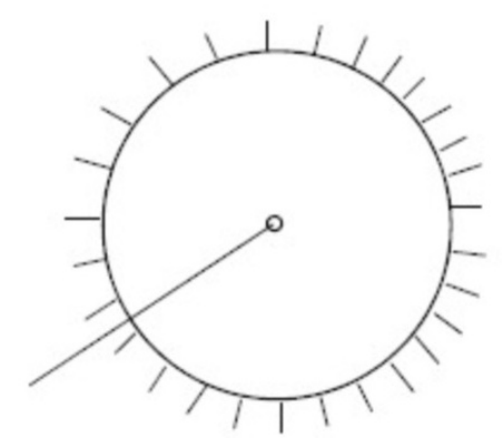

Figure 21. The ground state of the string state is obtained in the functional integral by propagating during an imaginary time span. If this time span is taken to be long, e.g. go to infinity, the dominantly surviving string state is the ground state.

$\theta(I)=2 \pi \cdot \frac{I}{N}$ and then we put (say)

$$
J_{R}^{\mu}(I) \stackrel{\text { ident. }}{=} \phi^{\mu}\left(\exp \left(i 2 \pi \frac{I+1}{N}\right)\right)-\phi^{\mu}\left(\exp \left(i 2 \pi \frac{I-1}{N}\right)\right)
$$

for all even $I$. Because of our technique of only taking the even objects as truly existing and being directly used in the second quantized description in our Hilbert space we only use the even $I$ values here.

Here the complex numbers exp $i 2 \pi \frac{I \pm 1}{N}$ refers to that the disk-shaper region A for the functional integral is imbedded into the complex plane as the unit disc (and so the norm unity numbers lie on the edge of the disc.)

The reader can relatively easily see first that the functional integral comes to depend on the object $J^{\mu}$ variables in a "Gaussian" (exponential of a quadratic expression in these $J^{\mu}(I)^{\prime} s$ ) way and then secondly that the "fluctuations" in the various Founrier resolution coefficients of these $J^{\mu}(I)^{\prime} s$ as a function of the angle $2 \pi \frac{I}{N}$ become the same as these fluctuations as estimated e.g. in our article on the mass spectrum of the string in our object-formulation [44].

But the easiest way to see that the functional integral gives the wave function is by thinking of producing the ground state by propagating during an imaginary time span. If this time span is taken to be long, go to infinity, the dominantly surviving string state will be the ground state.

This is illustrated above by the figure 21 .

Now we have four external particles giving rise to four such wave functions which can be written by means of discs or half-finite region functional integrals.

We shall mark the objects sitting along the edges of these discs by the symbols on figure 21.

Having in mind that we can as well as a disc use the complement of a disc depicted as figure 22 .

The meaning of the region is that outside a disc as the 2-dimensional region we can figure out a way to match the regions of objects marked with corresponding symbols, even in such a way that all four discs or complements lay on two layers in the complex 


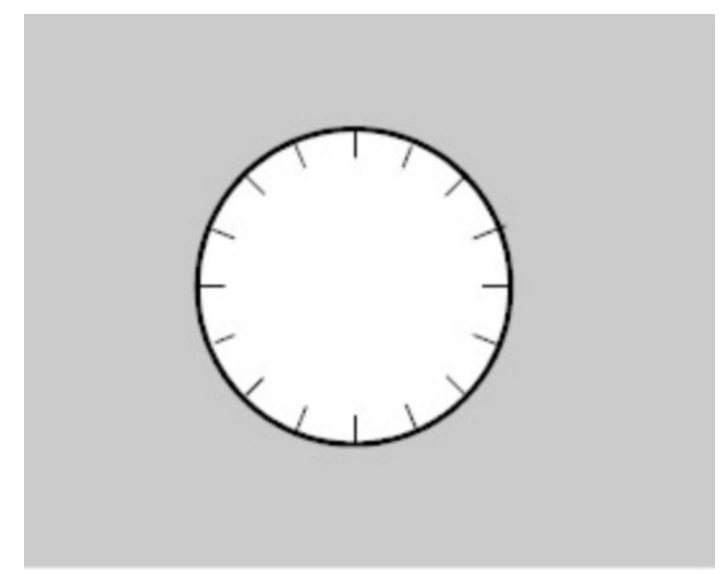

Figure 22. The complement of disc. Four external particles that give rise to four wave functions. They are written by means of discs or half-finite region functional integrals. The objects sit along the edges of the discs. We can use as well as disc's complement of a disc. In fact the region outside the disc is the two dimensional region. On this figure we have drawn two layers with the four discs put in as discs and complements.

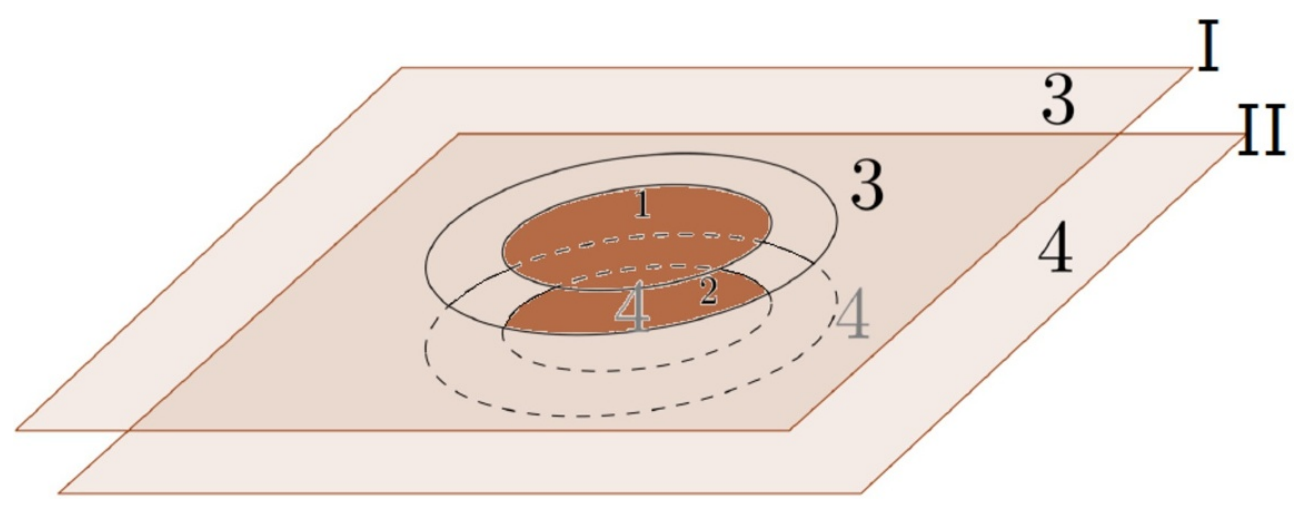

Figure 23. The two lines structure. Layer first one is denoted I while the second is II. These two layers are connected.

plane/Riemann sphere. In fact we shall put them so that 1 and 2, i.e. the incoming particles/strings have their wave functions represented by (proper) discs -lying in the two layers respectively -while the outgoing strings 3 and 4 are represnted by complements of also unit discs lying correspondingly by in the two layers over the complex plane.

In this way we get the whole complex plane covered doubly, one representative in each layer all over. To be definite we can choose to put in the first layer, called I, the disc for string 1 and the complement of the disc for string 3 .

We put them so that the common edge for these two regions just placed side by side (on the unit circle). Since by our assumption of all four strings having the same longitudinal momenta $P_{K}^{+}$so that $P_{1}^{+}=P_{2}^{+}=P_{3}^{+}=P_{4}^{+}$. We easily saw that

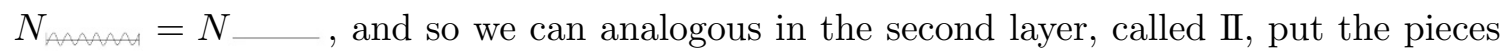
marked with — in the edges for 2 and 4 just covering the same part of the unit circle (just now on layer II) as the piece marked connecting 1 and 3 . 


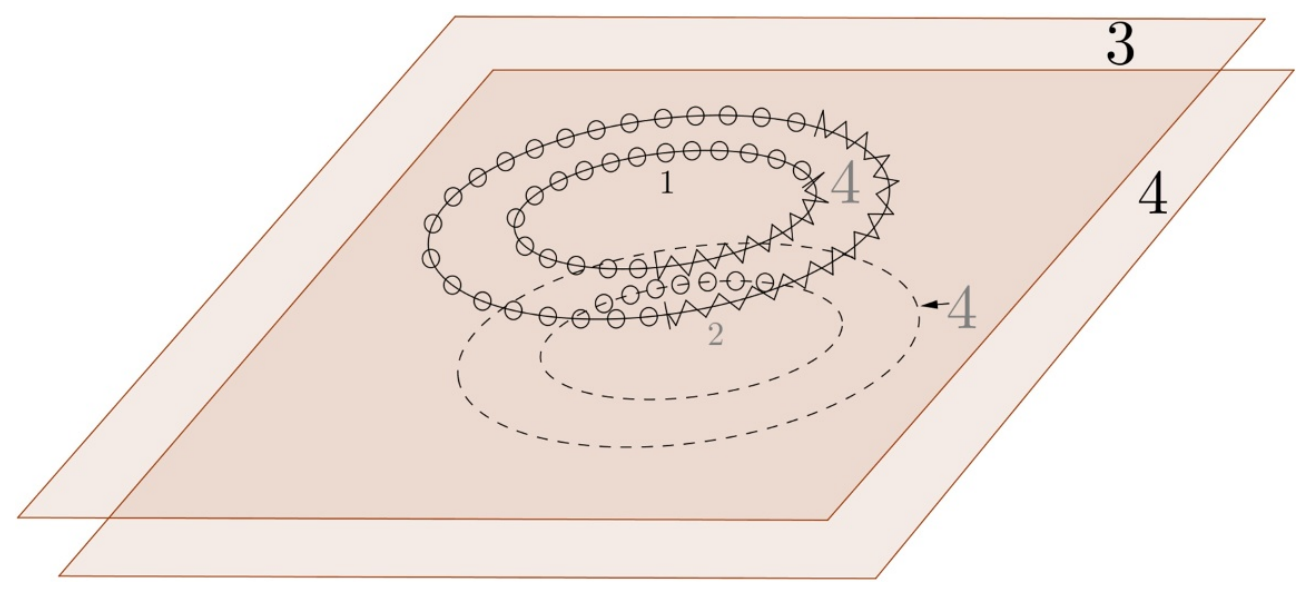

Figure 24. Here we have drawn the markings $\ominus 0000$, and along the edge of the complement 3 and along the pieces of edge for respectely $\ominus 0000$ on disc 2 and

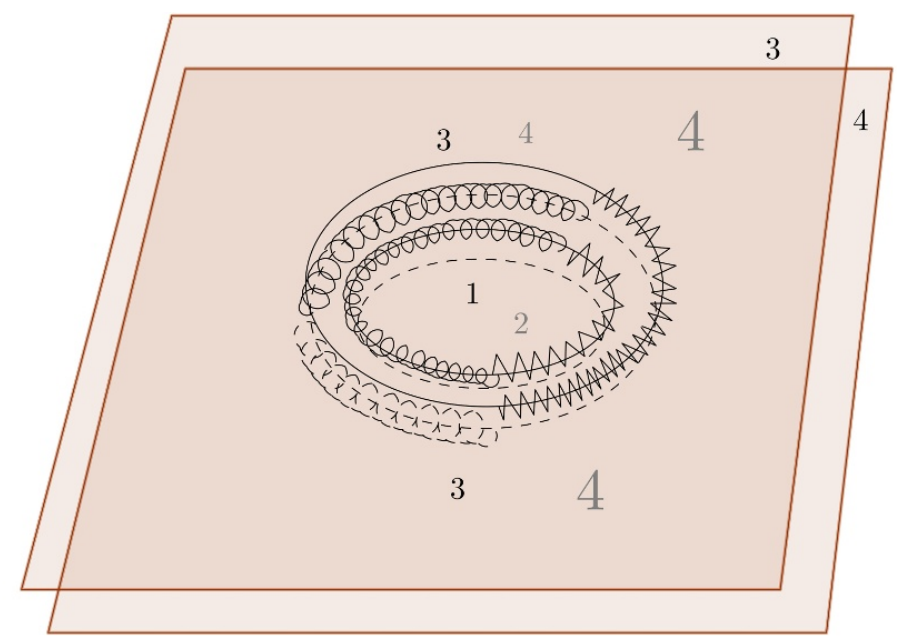

Figure 25. On this figure we show in a similar way the edge-decorations on the disc 1 marked and and the corresponding pieces of the edges of the complements 3 and 4.

In this placement of these discs and complements of discs one easily sees that by a simple cut running along the remaining part of the unit circle, (a cut along which layer I is continued into II and oppositely,) we achieve to continue disc for 1 into the complement of disc for 4 across the piece of unit circle now marked Analogously the disc for 2 gets continued along the $\ominus 000 \ominus$ edge across the cut into the complement of disc associated with string 3 .

The cut connecting I with II and oppositely must have a branch point in each of its two ends. About we manage to realize in the two-layered complex plane all the four gluings corresponding to objects in initial and final states being identified.

The gluing together of the regions for the functional along the curves where we identify objects in and outgoing states is supposed to lead to just the functional integral over the composed region (union of the glued together regions.) 


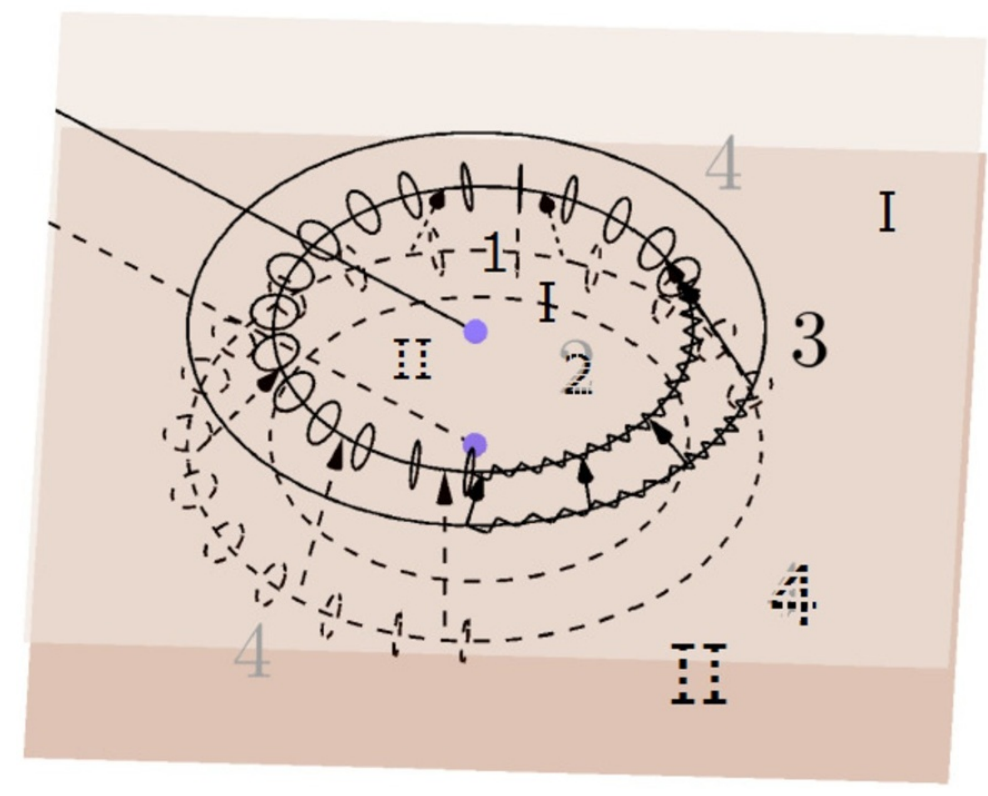

Figure 26. On this figure we have drawn arrows to represent the gluings, but to avoid making the figure incomprehensively complicated we left out the arrows. That should have connected disc 2 with complement 3 by arrows crossing in between the arrows connecting disc 1 with complement 4 both marked $\ominus 000 \ominus$. Similarly we left out the arrows that should have connected disc 2 and complement 4 both in the second layer II.

The full functional integrals with boundary conditions from 26-momentum inlets in the centers of the discs or in the infinity in the case of the complement of a disc becomes the overlap under the specific way of identifying objects in the initial and the final state.

The full overlap is therefore a sum over all the ways of identifying the objects of the overlaps under the specific identificaitons.

But we have made the approximation of only considering significant the identification patterns with the lowest number of jumps, where neighboring objects do not follow each other into the next cyclic chain.

Now we shall to evaluate the functional integral for a given identification of objects make use of that modulo the anomaly - to which we shall return - the value of the functional integral is invariant under conformal transformations of the two-dimensional region associated with this functional integral.

The most important step in the calculation is now to by a conformal transformation map the double layered complex plane/or better Riemann sphere into a single layered one. Very suggestively this shall be done by a square root type of analytic function, because a square root ambiguity gives two possible values.

Then we can use a complex $z$ give two different result values $f\left(z_{I}\right)$ and $f\left(z_{I I}\right)$ depending on which layer I or II we imagine the complex number $z$ to be.

Layer I drawn full —. . Layer II drawn punctured -.-.-. . All the four unit circles really coincide. It is only drawing technique they differ. Edges of 1 and 3 to be identified are on layer I. 
Now we shall seek to map our two layered representation of the two dimensional surface on which the functional integral is to be calculated by an analytical/conformal map into a one-layered one. To get inspired to what map $f$ say to choose we shall at first look at the situation near the two branch points. Imagining a little "circle" meaning a curve in a distance $\epsilon$ ( $\epsilon$ small) from the very branch point we see that it get actually the circumference $4 \pi \epsilon$ rather as a usual circle having only $2 \pi \epsilon$.

This implies that the function performing the map $f$ should have at square root singularity a square root like behavior near these two branch points.

Denoting the variable in the complex plane $z$ we get to each complex number $z$ two sheets with the two points on the full two-layered surface $z_{I}$ and $z_{I I}$. Here the $z_{I}$ is the point on sheet I and $z_{I I}$ the one on sheet II.

In this notation we can write down the attachment points $a_{i}(i=1,2,3,4)$ for the four external points:

$$
\begin{aligned}
& a_{1}=O_{\mathrm{I}} \\
& a_{2}=O_{\mathrm{II}} \\
& a_{3}=\infty_{\mathrm{I}} \\
& a_{4}=\infty_{\mathrm{II}} .
\end{aligned}
$$

Denoting the $z$-numbers corresponding to the branch points as $b_{A}$ and $b_{B}$ the need for square root behavior means that near these branch points we shall have

$$
f \widetilde{\propto} \pm \sqrt{z-b_{i}} \quad i=A, B
$$

or

$$
f \approx \frac{1}{ \pm \sqrt{z-b_{i}}}
$$

Here as well as when we finally choose $f$ it is to be understood that the sign ambiguity is to be resolved differently on the two sheets I and II (for the same $z$ ). At other places singularities of $f$ should rather be avoided. Actually the reader may easily see that the function

$$
f(z)= \pm \sqrt{\frac{z-b_{A}}{z-b_{B}}}
$$

is a good proposal for the transformation and that with appropriate sheet dependence of the \pm we get indeed a mapping into a single layer all covering Riemann sphere(being the image of $f$ ). Indeed we could check that $f$ maps the two layered twodimensional region (for the functional integral) surjectively (i.e. onto) to the Riemann sphere (with variable $f$ ) by constructing the inverse i.e. $z$ as function of $f$ : this inverse is gotten by the equation

$$
f^{2}=\frac{z-b_{A}}{z-b_{B}}
$$

meaning

$$
\left(b_{B}-z\right) f^{2}=z-b_{A} \quad \Longrightarrow \quad z=\frac{b_{B} f^{2}+b_{A}}{1+f^{2}}
$$


Under this map $f$ it is easily seen that the attachment points $a_{1}, a_{2}, a_{3}$ and $a_{4}$ are mapped into

$$
\begin{array}{ll}
f\left(a_{1}\right)=\sqrt{\frac{b_{A}}{b_{B}}} ; \quad f\left(a_{3}\right)=1 \\
f\left(a_{2}\right)=\sqrt{\frac{b_{A}}{b_{B}}} ; \quad f\left(a_{4}\right)=-1
\end{array}
$$

Remembering that the branch points lie on the unit circle in the $z$-plane

$$
\left.\left|b_{A}\right|^{2}=\left|b_{B}\right|^{2}=\right] 1
$$

we see that indeed all four images of the attachment points are also on the unit circle (but now in the $f$-plane)

$$
\left|f\left(a_{i}\right)\right|^{2}=1 \text { for } i=1,2,3,4
$$

Note immediately that such positions are just like Koba-Nielsen variables [54] for a four point Veneziano amplitude.

\subsection{The philosophy of counting}

The major purpose of these rather detailed calculations is to obtain the Veneziano amplitude correctly not only by having the right external momentum dependence but also has the correct form with respect to 26-momentum independent factors, only depending on the integration dummy. In order for such an ambition level to make sense we must start also from a well defined integration measure or rather we prefer to start from a well defined summation over a number specifying how many objects are common for some couple of external strings. But such a summation over a number of objects we have already developed above in section 5.1 especially eq. (5.14).

We have a little freedom not yet used to orient the discs and the complements, which we can use to arrange that the two branch points $b_{A}$, and $b_{B}$ become each others complex conjugate. In fact we may take

$$
b_{A}=e^{i \delta} ; \quad b_{B}=e^{-i \delta}
$$

with $\delta$ being a real angle. it is then easily seen that the number say

$$
N_{\text {AanAH }} \propto \delta
$$

so that the counting measure $\sum_{N} \sim d N_{n}$ becomes proportional to the integral over this angle $\delta$

$$
\sum_{N} \sim d N_{\triangle A M A} \propto d \delta
$$




\section{Evaluation of the integrand}

\subsection{Evaluation of the integrand}

Coming to the actual evaluation we must first face the problem that obtaining the wave function by a huge imaginary propagation leaves us with an arbitrary and divergent normalization.

This divergence pops up by our need to led in at the attachment of the external particles/strings via small $\epsilon$-radius discs.

Then the divergence shows up by the functional integral coming out in first approximation as the exponent of the classical action getting terms proportional to $\log \epsilon$ into this classical action. However, since we shall not be so ambitious as to calculate the absolute normalization of the Veneziano amplitude it should be enough to just keep this $\epsilon$ cut off the same for all the contribution to the Veneziano amplitude. Especially we should keep our $\epsilon$ 's for the four external particle attachments fixed under the summation over the different numbers of objects exchanged say between 1 and 4 . That is to say we must keep the $\epsilon$ 's constant while varying the integration variable $\delta$. The terms proportional to the $\log \epsilon_{i}(i=1,2,3,4)$ are a priori expected to be also proportional to $p_{i}^{\mu} p_{\mu i}$, whereas terms involving the inner products of different external 26-momenta will be convergent.

Taking these squares $p_{i}^{\mu} p_{\mu i}$ to be just the masses squared

$$
p_{i}^{\mu} p_{\mu i}=m_{i}^{2}
$$

and using that the mass of a mass-eigenvalue of a string state is just a constant, we see that these divergences $\propto \log \epsilon_{i}$ are in principle not so severe because they only give constant factors to the over all amplitude, which we anyway give up calculating.

If we want to get the formula for the $B(-\alpha(t),-\alpha(u))$ written in terms of the usual integration variable

$$
B(-\alpha(t),-\alpha(u))=\int_{0}^{1} X^{-\alpha(u)-1}(1-X)^{-\alpha(t)-1} d X
$$

If we identify the channel $1+\overline{4} \rightarrow \overline{2}+3$ as the $t$-channel and $1+\overline{3} \rightarrow \overline{2}+4$ as the $u$-channel, while $1+2 \rightarrow 3+4$ is the $s$-channel, then the integration variable for this expression $\int X^{-\alpha(u)-1}(1-X)^{-\alpha(t)-1} d X$ shall be the anharmonic ratio that goes to zero in the situation when the $u$-channel incoming $z$-variables approach each other. In fact we must take

$$
X=\frac{\left(z_{1}-z_{3}\right)\left(z_{2}-z_{4}\right)}{\left(z_{4}-z_{3}\right)\left(z_{2}-z_{1}\right)}=\frac{z_{1}-z_{3}}{z_{4}-z_{3}}: \frac{z_{1}-z_{2}}{z_{4}-z_{2}}
$$

where we have chosen the denominator so as to $X \rightarrow 1$ when the $z$-corresponding to say the incoming strings $1+\overline{4}$ approach each other.

In the analogue model terminology we imagine currents proportional to the 26momentum $p_{i}^{\mu}$ to be pumped in an $\epsilon$-disc at the point $z_{i}$.

The external 26-momentum conservation will allow these currents to flow in a conserved way. The current running in at $z_{i}$ will if it just runs to infinity symmetrically by the in the model assumed specific resistance $2 \pi \alpha^{\prime}$ (we shall use $2 \pi \alpha^{\prime}$ rather than $\pi \alpha^{\prime}$ because 
we work with the double lead to a potential at the position $z$ in the Riemann sphere $\frac{1}{2 \pi} \cdot 2 \pi \alpha^{\prime} \ln \left|z-z_{i}\right|=\frac{\alpha^{\prime}}{2} \ln \left|z-z_{i}\right|$. Using this the total energy production rate in this analogue model would be

$$
\frac{1}{2} \sum_{\substack{i, j \\ i \neq j}} p_{i}^{\mu} p_{j \mu} \ln \left|z_{j}-z_{i}\right| \quad \text { (the } j=i \text { term diverge) }
$$

and so the exponential of this "heat production rate" becomes

$$
\prod_{i, j}\left(\frac{\left|z_{j}-z_{i}\right|}{\epsilon_{i}}\right)^{-\alpha^{\prime} p_{i}^{\mu} p_{j \mu}} \cdot \prod N_{i}
$$

But these divergent factors although constant as functions of the external momenta are not constant as function of the $z_{i}$-variables of say as function of the anharmonic ratio $X$.

Remembering that the normalization factor for the $i$ th external particle is

$$
N_{i}=\epsilon^{\alpha^{\prime} m_{i}^{2}}=\epsilon^{\alpha^{\prime} p_{i}^{2}}
$$

and the 26-momentum conservation

$$
p_{1}^{\mu}+p_{2}^{\mu}=p_{3}^{\mu}+p_{4}^{\mu}
$$

or in an all ingoing notation

$$
p_{1}^{\mu}+p_{2}^{\mu}+p_{3}^{\mu}+p_{4}^{\mu}=0
$$

we recognize that $\epsilon_{i}$ just appears to the power

$$
\text { "power of } \epsilon_{i} "=-\alpha^{\prime} p_{i}^{\mu} \sum_{j \neq i} p_{j \mu}+\alpha^{\prime} p_{i}^{2}=0
$$

and thus there is really no dependence on these cut off $\epsilon_{i}$.

From the definition

$$
X=\frac{z_{1}-z_{3}}{z_{4}-z_{3}}: \frac{z_{1}-z_{2}}{z_{4}-z_{2}}
$$

and we get

$$
\begin{aligned}
1-X & =\frac{\left(z_{4}-z_{3}\right)\left(z_{2}-z_{1}\right)-\left(z_{1}-z_{3}\right)\left(z_{2}-z_{4}\right)}{\left(z_{4}-z_{3}\right)\left(z_{2}-z_{1}\right)} \\
& =\frac{z_{4} z_{2}+z_{3} z_{1}-z_{1} z_{2}-z_{3} z_{4}}{\left(z_{4}-z_{3}\right)\left(z_{2}-z_{1}\right)} \\
& =\frac{\left(z_{3}-z_{2}\right)\left(z_{1}-z_{4}\right)}{\left(z_{4}-z_{3}\right)\left(z_{2}-z_{1}\right)} .
\end{aligned}
$$

Using in the $p_{1}+p_{2}+p_{3}+p_{4}=0$ notation

$$
\begin{aligned}
s & =m_{1}^{2}+m_{2}^{2}+2 p_{1} \cdot p_{2} \\
& =m_{3}^{2}+m_{4}^{2}+2 p_{3} \cdot p_{4} \\
t & =m_{1}^{2}+m_{4}^{2}+2 p_{1} \cdot p_{4} \\
& =m_{2}^{2}+m_{3}^{2}+2 p_{3} \cdot p_{2} \\
u & =m_{1}^{2}+m_{3}^{2}+2 p_{1} \cdot p_{3} \\
& =m_{2}^{2}+m_{4}^{2}+2 p_{2} \cdot p_{4}
\end{aligned}
$$


and thus

$$
s+t+u=m_{1}^{2}+m_{2}^{2}+m_{3}^{2}+m_{4}^{2}
$$

to replace

$$
p_{1} \cdot p_{2}=\frac{1}{2}\left(m_{3}^{2}+m_{4}^{2}-t-u\right) .
$$

We obtain

$$
\begin{aligned}
&\left(\Pi N_{i}\right) \cdot \prod_{i, j}\left(\frac{\left|z_{i}-z_{j}\right|}{\epsilon_{i}}\right)^{-\alpha^{\prime} p_{i}^{u} \cdot p_{j u}} \\
&=\prod_{\substack{i, j \\
i \neq j}}\left|z_{i}-z_{j}\right|^{-\alpha^{\prime} p_{i}^{u} \cdot p_{j u}} \cdot\left(\prod_{\substack{i, j \\
i \neq j}}\left|z_{i}-z_{j}\right|^{-\alpha^{\prime} m_{i}^{2}}\right) \\
&= \prod_{\substack{(i, j) \\
\text { with } i \neq j \text { but each } p}}\left|z_{i}-z_{j}\right|^{-2 \alpha^{\prime} p_{i}^{u} p_{j u}-\alpha^{\prime}\left(m_{i}^{2}+m_{j}^{2}\right)} \\
&= \prod_{\substack{(i, j) \\
\text { one order }}}\left|z_{i}-z_{j}\right|^{\alpha^{\prime}\left(p_{i}-p_{j}\right)^{2}} \\
& \cdot\left|z_{1}-z_{3}\right|^{-\alpha^{\prime} u} \cdot\left|z_{2}-z_{4}\right|^{-\alpha^{\prime} u} \\
& \cdot\left|z_{1}-z_{2}\right|^{-\alpha^{\prime}\left(-t-u+m_{3}^{2}+m_{4}^{2}\right)} \cdot\left|z_{3}-z_{4}\right|^{-\alpha^{\prime}\left(-t-u+m_{1}^{2}+m_{2}^{2}\right)} \\
& \cdot\left|z_{1}-z_{4}\right|^{-\alpha^{\prime} t} \cdot\left|z_{2}-z_{3}\right|^{-\alpha^{\prime} t} \\
&= X^{-\alpha^{\prime} u}\left(1-X^{-\alpha^{\prime} t}\left|z_{3}-z_{4}\right|^{-\alpha^{\prime}\left(m_{1}^{2}+m_{2}^{2}\right)} \cdot\left|z_{1}-z_{2}\right|^{-\alpha^{\prime}\left(m_{3}^{2}+m_{4}^{2}\right)}\right.
\end{aligned}
$$

\subsection{What is required to finish Veneziano model?}

To get the last bit of the way to obtain all three terms in the four point Veneziano model in our object scheme we have to obtain a definite counting of the number of ways of identifying or better bringing in correspondence the objects in the initial state with those in the finial state.

In our previous article we used as a combined gauge or parameter $\tau_{R}$ choice and discretization to impose the condition that each object has it $J^{+}$, a special component of its $J^{\mu}$ take a specific value

$$
J^{+}=\frac{a \alpha^{\prime}}{2}
$$

for all the objects.

This "longitudinal momentum" (or "longitudinal $J^{\mu ")} J^{+}$(which is essentially the + component of momentum of the object) is defined

$$
J^{+}=J^{0}+J^{25}
$$

where $J^{25}$ is the infinite momentum frame direction. If, and that is indeed true since classically

$$
\left(J^{\mu}\right)^{2}=0,
$$

the $J^{\mu}$ is "on shell" as a momentum then

$$
J^{+} \geq 0 .
$$


But if we allow "energy" $J^{0}$ to be negative then also $J^{+}$is not guaranteed to be positive.

As long as the $J^{+}$'s are guaranteed positive as we used in previous paper, then one can devide the positive +-momentum into $J^{+}$'s all being some given positive number

$$
J^{+}(I)=\frac{a \alpha^{\prime}}{2} .
$$

But now in order to be allowed to hope for obtaining all the 3 terms in the full Veneziano amplitude we must accept negative $J^{+}$'s.

So we must choose a gauge or parametrization and discretization choice that is more liberal with respect to allowing negative $J^{+}$too. The obvious suggestion is that in constructing the dicretization we first imagine dividing the cyclic chians corresponding to the strings into pieces with negative $J^{+}$'s and pieces with positive $J^{+}$'s. In order that this shall be nice it should be so that the $\dot{X}_{R}^{\mu}\left(\tau_{R}\right)$ that is proportional/essentially the same as $J^{\mu}$ is so smooth that the sign of $\dot{X}_{R}^{+}\left(\tau_{R}\right)$

$$
\operatorname{sign}\left\{\dot{X}_{R}^{+}\left(\tau_{R}\right)\right\}
$$

is constant over intervals of reasonably large size.

With some - may be a bit vague - continuity assumption these large intervals of fixed signs $\operatorname{sign}\left\{\dot{X}_{R}^{+}\left(\tau_{R}\right)\right\}$ is justified. Also one has in this spirit also the assumption that the pieces of a cyclic chain in an initial state string going in the overlap into a given final state cyclic chain will consist dominantly of very few pieces. It shall with highest weight be connected to one connected piece only. In the scattering the negative energy $J^{0}$ or negative $J^{+}$pieces have to for say an initial cyclic chain must either annihilate with positive piece in another incoming cyclic chain or go on as a negative piece in the final state.

\section{Dominant term in $1+2 \rightarrow 3+4$ scattering}

\subsection{Dominant term in $1+2 \rightarrow 3+4$ scattering}

Mandelstam has diagrams describing his contribution to the Veneziano amplitude in which two strings are interacting by their ends (contrary to ours which rather interact the intuitively most likely way by crossing each other on a random point somewhere on the string ) and then t propagates them in what is effectively imaginary time. Some such imaginary time before they again split into two strings. It we now say that hidden in this imaginary time propagation can be hitten a partial annihilation, it means that Mandelstam has something that very likely is partial annihilation of pieces of one of the incoming strings with part of the other one. Translated to our cyclically ordered chain that could easily mean that Mandelstam has indeed in his picture what we take as the effect of having negative $P^{+}$. To say it shorter: Mandelstam has, in real physical sense, involved imaginary time development - while we only use it as a trick to make wave functions. Thus he also has this physically very strange thing that the strings interact with their END-points rather than what classically thinking is definitely more likely by the random points of the two incoming strings meeting. This means that he -Mandelstam- has a strange quantum effect treated by a very formal treatment with imaginary time included. So from our "more realistic" 


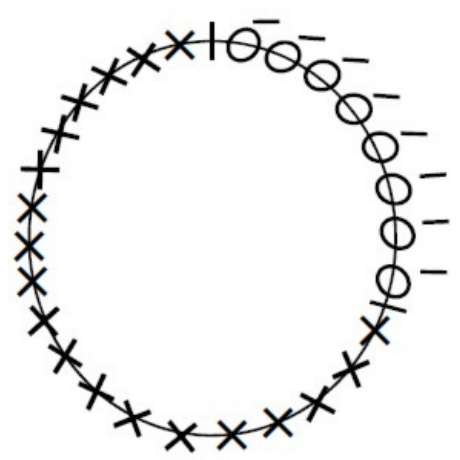

1

Figure 27. Dominant term in the case of the cyclic chains with a negative piece.

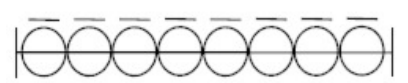

Figure 28. Negative part of pieces of string 1 .

point of view Mandelstam, and most string theorists work with some quantum tunneling as if it were a true physical description, and then sometimes one might get possibility for doing something - like getting all three terms-so that it looks physically done, but in reality might not be possible (with the assumed positive $P^{+}$). Further negative $P^{+}$case will be investigated in our forthcoming paper.

According to our somewhat vague continuity assumption the dominant term to a scattering amplitude should come from a system of houw pieces of the incoming cyclic chains going into the final state or annihilate in the most simple way. I.e. it is the system with fewest pieces involved. Let us see what is this dominant term in the case of one of the initial cyclic chains having a "negative" piece.

We put minuses to indicate the "negativeness" of a piece of the to string 1 correspinding "negative part" in the string 1 here denoted

The interesting case is when the piece $|\bar{\theta} \bar{O} \bar{O} \bar{\theta} \bar{O} \bar{O}|$ of negative cyclic chain in string 1 gets annihilated by a correspoinding piece in the string 2 cyclic chaing.

Under the assumption of as few pieces as possible the cyclic chain for string 2 should

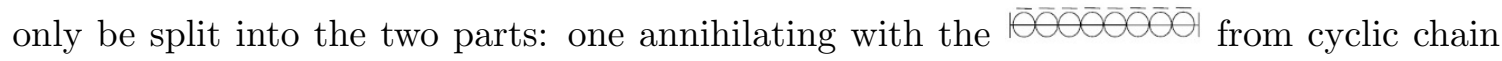
1 and one part continuing to just one of the final state strings, say string 3 , or rather its cyclic chain. So let us write the whole initial state cyclic chain as:

Now we have in the final state for the considered process

$$
1+2 \rightarrow 3+4
$$

also two open strings and therefore two cyclic chains 3 and 4 .

From the continuity assumption or assumption of fewest pieces dominating then all the $|x \times x \times x \times x \times|$ goes to one, say cyclic chain 4 , while the piece from $2 \mathrm{~m}$ continues in cyclic chain 3 . 


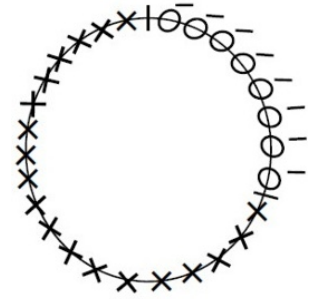

1

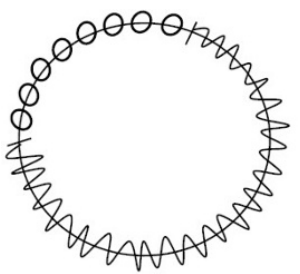

2

Figure 29. Incoming string 1 cyclic chain negative part and string 2 cyclic chain splits into the two parts: one annihilates with the negative piece from the cyclic chain 1 and one part of the final string e.g. string 3 cyclic chain
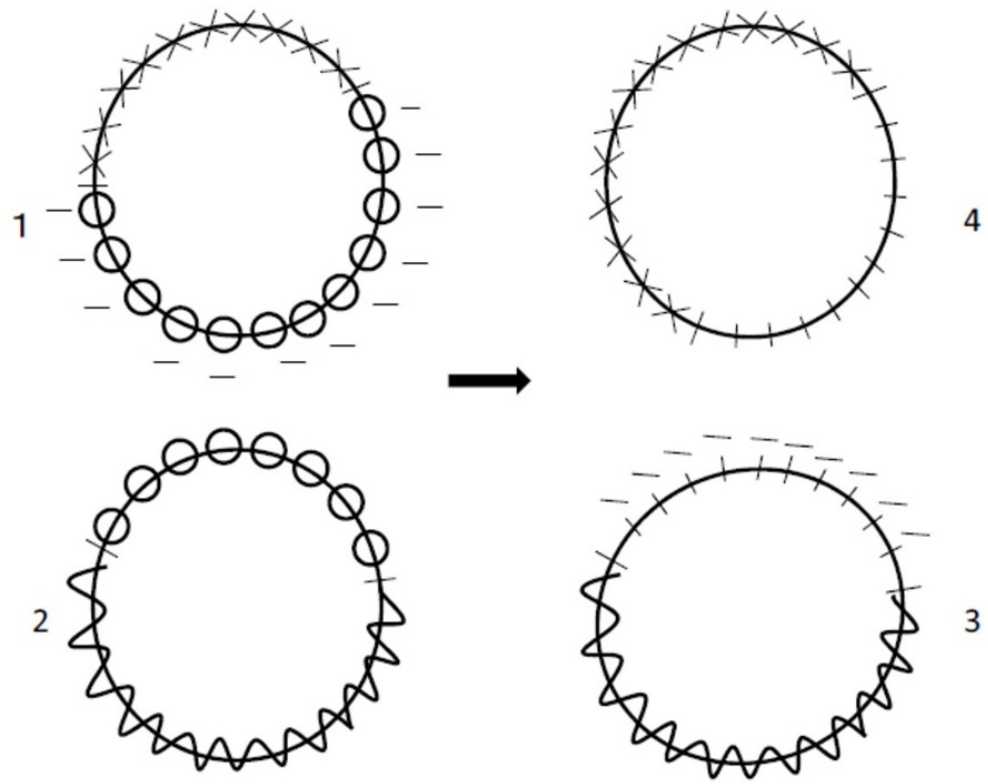

Figure 30. The analogue of figure 19 but now negative $P^{+}$(and thus energy) on the with - 's marked pieces of the cyclic chains for string 1 and string 3 .

Now the only way to have the rests of 3 and 4 dispences which is to let a piece from 3 and from 4 annihilate each other.

Finally we thus end up with the scheme represented by the figure 30 .

The "gauge or discretization condition" that we here choose is proposed:

$$
\begin{array}{ll}
\text { For positive pieces : } & J^{+}=\frac{a \alpha^{\prime}}{2} \\
\text { For negative pieces : } & J^{+}=-\frac{a \alpha^{\prime}}{2} .
\end{array}
$$

Then conservation of the $p^{+}$-component of the 26-momentum implies that the number of (even) objects in the positive pieces of the initial state minus those in the negative pieces, in our example

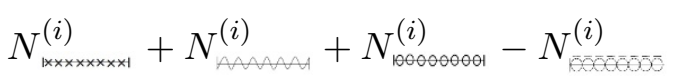




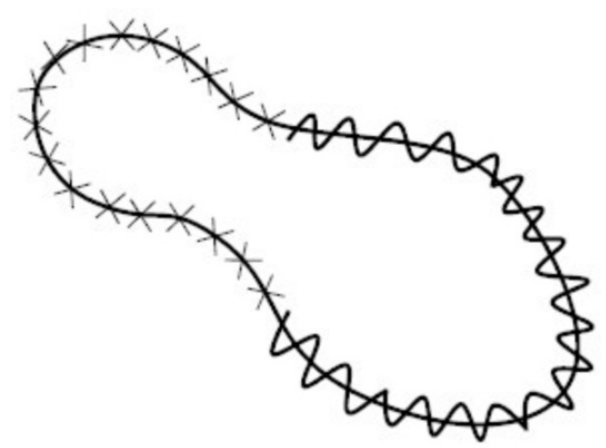

Figure 31. The important part of the set of the cyclically ordered chain of objects that really exists on the figure depicted as figure 31.

must be equal to the same quatity for the final state

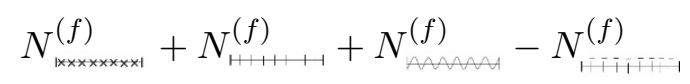

That is to say we must have

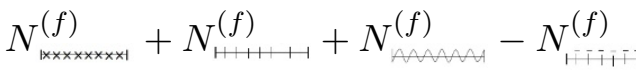

$$
\begin{aligned}
& =N_{1 \times x \times x \times x \times x \times 1}^{(i)}+N^{(i)}+N_{1000000001}^{(i)}-N_{0000053}^{(i)} .
\end{aligned}
$$

But noticing that in order to have an "annihilation" of a series of positive energy objects with a series of negative energy ones there should be equally many of them, we have

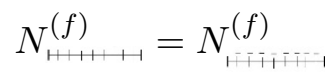

and

$$
N_{100000001}^{(i)}=N_{000005050}^{(i)}
$$

and that also for the correspondence of the objects in initial state with those in the final state requires

$$
N_{1 \times x \times x \times x \times x \mid}^{(f)}=N_{1 \times x \times x \times x \times x 1}^{(i)}
$$

and

$$
N_{\text {(f) }}^{(f)}=N_{\text {(i) }}^{(i)}
$$

We see that the equation (7.6) is trivially satisfied.

Thinking of the important part of the set of the cyclically ordered chains of objects.

The quantity that has to be summed over is the amount of objects really existing which belong to string 1 or equivalently string $4^{|x \times x \times x \times x \times|}$ as contrary to the rest which belongs to string 2 or equivalently to string 3 . The sum of the numbers of objects in these two classes, the 1 or $4^{|x \times x \times x \times x \times|}$ and the 2 or $3 \mathrm{~mm}$ is constant for given external 26 dimensional-momenta of the four strings

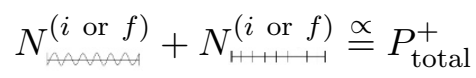


due to that this sum is proportional to the sum of the "longitudinal" momenta $p_{1}^{+}$and $p_{2}^{+}$of string 1 and string 2 . So for a set of fixed external momenta for which we want to evaluate the overlap or the scattering amplitude the only physically existing variation in the

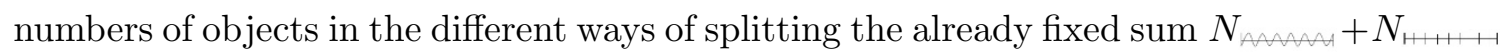
into its two parts. Each choice of a part should be weighted with no extra weight, when we just compare one positive integer value for $N$ with another positive integer value for $N$

\subsection{Problems in getting the weight of Veneziano integral easily}

To make the derivation of the Veneziano model from our object-based string field theory so easy as possible while still using the "gauge" in which $J^{+}= \pm \frac{a \alpha^{\prime}}{2}$, which could be called $\operatorname{IMF}(=$ infinite momentum frame) gauge, we shall for each of the three terms which we hope to obtain in full Veneziano model choose a different condition specifying the Lorentz frame shall use.

We want to arrange that when say there are no more objects left on string 1 we truly reached the end of the chain of possibilities to be summed over. Essentially we want the reach of zero or run out of objects to occur "simultaneously" for say both string 1 and some other string that runs out.

Let us be more precise and first notice as one varies the numbers of objects $N_{\text {and }}$,

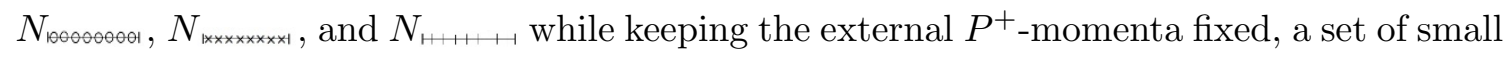

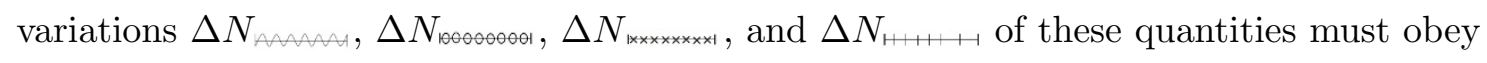

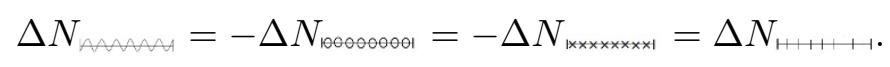

For fixed external $P^{+}$'s we has that

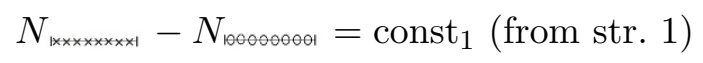

$$
\begin{aligned}
& N_{1000000001}+N_{\text {AnAS }}=\text { const }_{2} \text { (from str. 2) } \\
& N_{\text {AnAMH }}-N_{\text {HI1 H }}=\text { const }_{3} \text { (from str. 3) }
\end{aligned}
$$

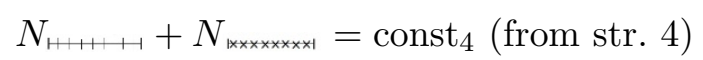

These numbers of objects, negative $J^{+}$of positive $J^{+}$, must be positive and thus they must run in the intervals

$$
\begin{aligned}
& 0 \leq N_{\text {and }} \leq 4 P_{2}^{+} / a \\
& 0 \leq N_{\text {H, म H }} \leq 4 P_{4} / a
\end{aligned}
$$

Now we want to make the boundaries so simple as possible by letting the ranges of the different $N$ 's be the same, otherwise we get the problem that since only one $N$ can be varied independently we could not fill out both ranges. Indeed we thus want to make the two ranges correspond to each other and thus especially need that the length of the interval for $N_{\text {w }}$ which is $4 P_{2}^{+} / a$ be the same as that for $N_{100000001}$, namely $4 P_{4}^{+} / a$. Thus we are driven to -for simplicity- to claim that we shall suggest to arrange by a Lorentz transformation that

$$
P_{2}^{+}=P_{4}^{+}
$$


If we decide that also $N_{100000000}$ and $N_{H}$ shall run over the same interval, we must let $N$ and $N_{\text {}}$ start simultaneously at zero and similarly also $N_{1}$ and $N_{\text {r }}$ should start at zero simultaneously. So at the end we are driven towards

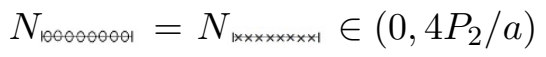

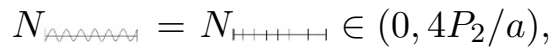

these two couples running though opposite to each other. The first of these two equations imply that $P_{1}^{+}=0$ and the second one that $P_{3}^{+}=0$. In conclusion we suggest that to avoid troubles with not being allowed to sum over just one interval we shall restrict our consideration to the special situation achievable in principle a Lorentz transformation such that

$$
\begin{aligned}
P_{1}^{+} & =P_{3}^{+}=0 \\
\text { and } \quad P_{2}^{+} & =P_{4}^{+} .
\end{aligned}
$$

With this special type of external momentum configuration we remark that we have in the cyclic chains for string 1 and string 3 just the same number of negative and positive $J^{+}$objects.

On the other hand we take the cyclic chains of strings 2 and 4 to have only positive $J^{+}$objects.

Analogously to the writing of the wave function in the case of only positive $J^{+}$objects as functional integrals, we shall also write the wave functions here. For strings 2 and 4 where we have only positive $J^{+}$objects, it is exactly as in the totally positive $J^{+}$case. But for strings 1 and 3, we have to ensure ourselves that we can just say that looking for transverse momentum the functional integral is just given by the number of objects no matter if they have positive $J^{+}$or negative $J^{+}$. This should be so because in the functional integral formally the different components of 26 momentum, i.e. different $\mu$, are completely decoupled.

Now, however, we discovered a little problem - the species doubler problem $[52,53]$ the herely associated nonorientation invariant "continuity condition".

If we shall indeed be able to have a piece of cyclic chain with positive $J^{+}=\frac{a \alpha^{\prime}}{2}$ cancel a piece with negative $J^{+}=-\frac{a \alpha^{\prime}}{2}$ then the continuity conditions for the "negative" and for the "positive" pieces must match so as to make cancellation possible. The requirement needed is that we can have a "positive" piece say with both odd and even $J^{\mu}$ 's for some component index $\mu$ be just opposite to those for a "negative" piece. Now, however, because of the non-orientation invariance it is very important how we decide to order the numbering $I$ along the two pieces that shoule cancel. To see how we need to require the orientations let us consider the following figure illustrating a cyclic chain in the Minkowsky space time which is interpreted as composition from two different cyclic chains, one of which has a "negative" piece.

Here the phantasy part for a pair of compensating pieces is shown in figure 32 where the orientation arrows have been put on so that the total can also be interpreted as the two separate cyclic chains. The point to be drawn from these figures is that provided we 


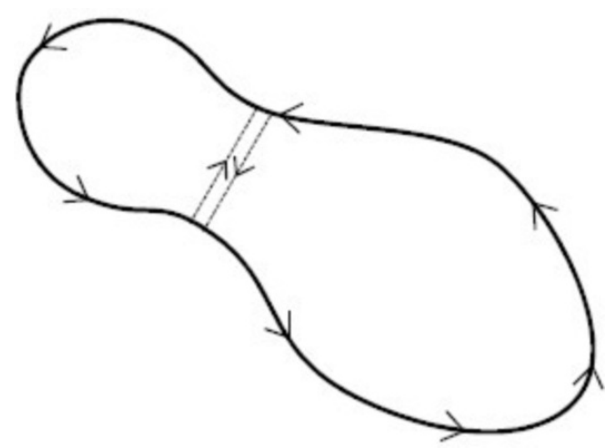

Figure 32. The figure shows how we need to require the orientations. The cyclic chain in the Minkowski space time. There should be considered: (a) There are negative and compensating parts indicated by dotted line. These parts are pair of compensating pieces. (b) The cyclic chain with positive parts denoted by solid line with positive parts.

insist on a simple cyclic ordering for our cyclic chains we must accept an opposite ordering of the two conpensating pieces. This in turn means that requiring for the "positive" pieces the usual "continuity condition" we are driven to take the opposite "continuity condition" for the "negative" pieces. In other words we are driven to:

For the number I odd case:

$$
\begin{gathered}
J^{\mu}(I+1) \approx-\Pi \alpha^{\prime}\left(\Pi^{\mu}(I+1)-\Pi^{\mu}(I-1)\right) \approx J^{\mu}(I-1) \\
\text { for } \quad J^{+}(I)=\frac{a \alpha^{\prime}}{2} \quad \text { i.e. positive }
\end{gathered}
$$

and

$$
\begin{array}{r}
J^{\mu}(I+1) \approx+\Pi \alpha^{\prime}\left(\Pi^{\mu}(I+1)-\Pi^{\mu}(I-1)\right) \approx J^{\mu}(I-1) \\
\text { for } \quad J^{+}(I)=-\frac{a \alpha^{\prime}}{2} \quad \text { i.e. "negative". }
\end{array}
$$

When going to the discussion using the complex plane the non-orientation invariant "continuity condition" can, as is rather easy, be considered a Caucy-Riemann condition for our $\phi^{\mu}$ in the functional integral. When we, as the technical trick, to produce the ground state via an imaginary $\tau$-time propagation "sneak in" conplex numbers our a priori real $\phi^{\mu}$ gets complex as say classical solution.

In any case if we under conformal transformations want to keep the continuity conditions undisturbed, we must take it that to the negative objects, $J^{+}<0$, corresponding regions in the two dimentional space for the functional integral have anti-analytic rather than analytic $\phi^{\mu}$ 's. Here we imagine that we associate with each section of negative $J^{+}$a behind it region in the two-dimensional region for the functional integral. In this way we divide up the two-dimensional region into pieces that should be conformally transformed only under respectively analytical and antianalytical maps in order that the "continuity conditons" be kept.

At the boarder line between the "negative" and "positive" regions the regions goes from analytical to anti-analytical, and a natural way to put it into the complex plane would be 


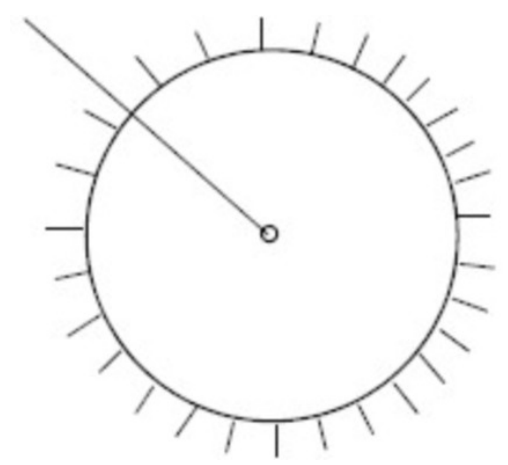

Figure 33. The wave functions for the strings 1 and 3 by the functional integral of following figure as a disc or a half infinite cylinder.

to give it a reflexion line here between the two regions. In this way like the strings 2 and 4 with only "positive" objects get their wave functions represented by function on a region being a half infinite cylinder or conformally equivalent disc (with the objects sitting on the edge and the momentum of the string entering in the centrum.)

Also the strings 1 and 3 get their wave functions reresented by functional integral of what is represented as a disc or half infinite cylinder.

Now these 4 figures for the four external particles/strings have to be glued together according to the rules of the identification of the initial and final states in terms of even object.

However, we can only glue together, to either annihilate or identity -from initial and final state- pieces of the edges of these discs provided the identified or annihilated pieces have the same numbers of objects.

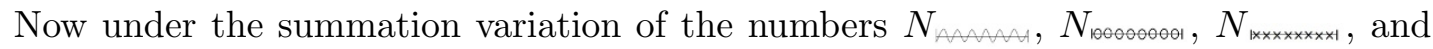
$N_{H+11+1}$, however the number of "negative" and "positive" objects on the edge of the disc for strings 1 and 3 varies.

So to bring e.g. the number $N_{1000000001}$ of "negative" objects on the cyclic chain for string 1 to annihilate with the corresponding $N_{100000000}$ "positive" objects on string 2 marked ${ }_{000} 00000$ in a way in which pieces of a unit circle match we must first make a conformal transformation of the disc for string 1 so that its edge for the objects marked on the a priori figure- $180^{\circ}$ rather becomes $2 \pi \frac{10000000 \text { or }}{4 P_{2}^{+} / a}$, namely the angle corresponding to the 10000000 marking of the disc for string2.

This conformal map must be an exponentiation with the power

$$
\frac{2 N_{1000000001}}{4 P_{2}^{+} / a}=\frac{2 N_{1000000001}}{N_{1000000001}+N_{\text {andi }}} .
$$

I.e. the variable say $y$ for the disc should be transformed

$$
y \rightarrow \xi=y^{\frac{2 N_{100000001}}{N_{1000000001}+N_{\text {and }}}}
$$

so that the unit circle parametrization by $\delta$ say as

$$
y=e^{i \delta}
$$




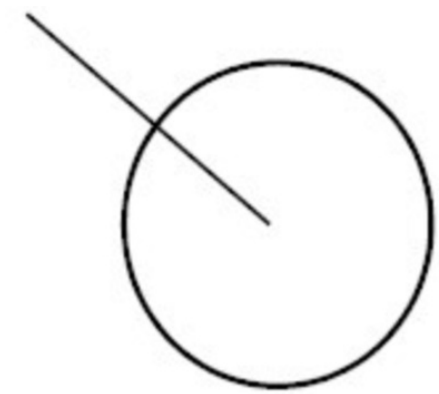

Figure 34. To get the wave functions of the strings 1 and 3 , we make a functional integral in a disc or half infinite cylinder figure 33 constructed described in the manner of the main text.

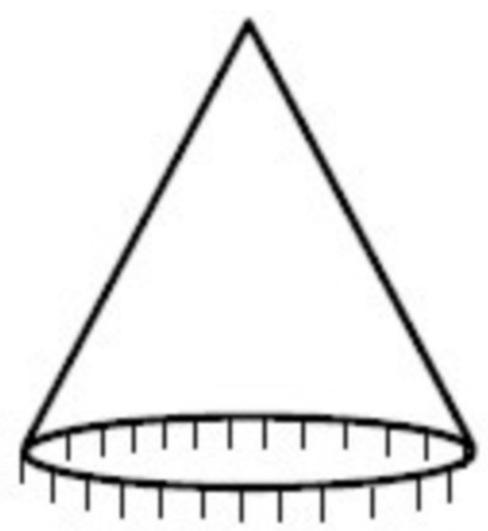

Figure 35. A half figure conformally transformed from figure 34. We can scale up or down by changing the length of the edge with the objects attached to be.

would be scaled by this factor $\frac{2 N_{1000000001}}{N_{1000000001}+N_{\text {and }}}$ or

$$
\delta \rightarrow \frac{2 N_{1000000001}}{N_{1000000001}+N_{\text {man }}} \cdot \delta .
$$

In this way we get the disc are transformed rather into a "hat".

Now with the lengths of the edges with the object attachments reduced or scaled up to be rather than of circle length $\pi$ of circle length $\frac{2 N_{100000001}}{N_{100000001}+N_{\text {m n }}} \cdot \pi$. But after such scalings of the edges for the folded discs for both string 1 and string 3 we can -nicely-glue the 4 previous discs, two of which are now hats, to actually topologically speaking one Riemann sphere.

Since a disc and the complement of a disc are conformally equivalent we can describe the result as the following a), b),..,f) of the appropriate gluing of the regions for the functional integral:

a) Put the string 2 disc as genuinely a unit disc into the complex plane.

b) Take the string 4 disc instead of as a genuine disc to be represented by the complement of a disc. Then the edges for string 2 and string 4 lie just on the same unit circle. But string 2 and string 4 have no pieces to be identified at all since 2 only have and 100000000 while 4 has only 
c) So we need to put the two hats produced with circular curve lengths respectively

$$
\begin{aligned}
& 1-\text { curvelength }=\frac{2 N_{100000001}}{N_{1000000001}+N_{\text {and }}} \cdot \Pi
\end{aligned}
$$

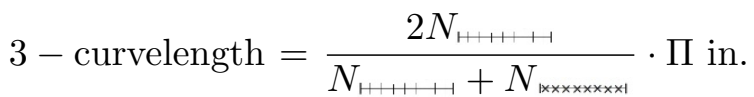

Since according to our restrictions (during the summation)

$$
N_{1000000001}=N_{1 \times x \times x \times x \times x \mid}
$$

and

$$
N_{\text {AAMAH }}=N_{\text {H11н }}
$$

we have to have 1 - curvelength +3 - curvelength $=2 \pi$ meaning that the curve lengths of the two "hats" just add up to $2 \pi$, the circumference unit circle seperating the disc for string 2 and the disc-complement for string 4 .

d) We shall attach the annihilation gluings. That is to say that for example the hat for 1 with its negative series 10000000 of objects to be attached to 2 .

e) Then the "positive" edge for 1 named ${ }_{x \times \times x \times \times \times x \mid}$ can extends straight into the equally named ${ }_{x \times \times \times x \times x \times 1}$ for the disc complement for 4 .

f) Analogously the hat for 3 should be complement for 4 along its "negative" piece. The positive part on the hat for 3 then can go straight into the also marked edge of the disc for 2 .

In the configuration just achieved the different 2-dimensional pieced have be put so that the functional integral variable $\phi^{\mu}$ should be analytic all over in all the up to 3 layers now being regions.

When we seek to construct a Riemann sphere or the like we still have the problem that along the internal separation lines in the hats 1 and 3, You may reflect the layers of the "negative" type.

\section{Conclusion and outlook}

We develop a new description for an arbitrary number of strings, a string field theory.

It is formulated in terms of a discretization into pieces - much like Thorn's string bits, but we do it for right and left movings - components and the then string bits of thorn are then called by us "objects".

These objects have dynmmics like free massless particles. That is to say they are decided by a quantum field theory of free massless particle. In momentum space they are static.

So nothing happens, even if the strings scatter! strings.

We have arguments that our model is really a transformation of theory for several 
As for deriving the Veneziano model, first with some troubles, but having negative even energy for the objects will presumably help to get the full Veneziano model (we missed two terms at first).

Also the spectrum we got o.k., except for a species doubler problem [52, 53].

(Apart from null sets) our string field theory should be just a rewriting of usual say string field theory.

The Hilbert space describing all the possible states in a string world is the Fock space of - either one or two - theories of massless noninteracting scalars (for the bosonic $25+1$ model).

Two massless free scalar theories/species of scalar particles for purely closed string theory, while only one when there are open strings.

But allowed states are restricted to obey - approximately — some "chiral" invariant continuity condition: this means that the stringyness only comes in via initial state conditions.

We think we have a new(novel) way of representing string theory, which because of being in some respects simpler could be helpful in understanding some aspects of string theory better.

Even if string theory should not turn out to be the final truth -as can still be the caseits abilities for providing a cut off are so good that alone in looking for cut off it may give inspiration.

It happens generally thinking to seek a cut off you easily get in the direction of the string theory, especially the aspect of not having any true interaction as is a trademark for our novel SFT model.

Our novel field theory deviates from usual ones - Kaku Kikkawa's or Witten's by including (a nul set of) of information less in its description of state of the world, i.e. of a set of strings present.

We have rewritten the information - the kept part - on a state of several strings into a state of something (more like particles), which we call "objects", to such a degree that one only sees the connection to genuine strings by quite a bit of complicated rewriting.

Our novel string field theory is genuinely nonperturbative theory. We should be able to redenve nonperturbative theory of string theories such as branes. Also so fan the background space time is flat. Next step will be taking non flat, e.q. pp-wave background.

Now if our string field theory (and string theory) is the theory of everything (TOE), we should be able to derive inflation theories in early universe: it may be one of the greatest challenge which we are planning to attack.

In very high energies such as Planck scale and/or string scale, we may be able to investigate truly new physics, for instance, studying supersymmetric particles.

\section{Acknowledgments}

The authors thank K. Murakami, K. Sugiyama, M. Sakaguchi and Y. Sekino for their useful comments. 
One of us (H.B.N.) acknowledges the Niels Bohr Institute for allowance to work as emeritus. M. Ninomiya acknowledges Yukawa Institute for Theoretical Physics, Kyoto University and Osaka city University, Advanced Mathematical Institute supporting this work. the Niels Bohr Institute and the Niels Bohr International Academy for giving him very good hospitality during his stay. M.N. also acknowldges the present research is supported in part by the JSPS Grant in Aid for Scientific Research No. $15 \mathrm{~K} 05063$.

H.B.N. thanks to the Bled Conference participants, organizers and Matiaz Breskov for finantial support to come there where many of the ideas of this work got tested.

\section{A What is the rough Dirac sea?}

In a free theory of second quantized fermions it is well known that the negative energy single fermion states are all filled while the positive energy single fermion states are all empty.

When there are interactions between such fermions or with other fields the ground state is no longer so simple. The vacuum is in this case rather a superposition of a lot of free energy states of the second quantized theory, a lot of which have empty single fermion negative energy states, or filled positive single fermion states.

This is analogous to that in a peaceful sea there is water for negative height and air for eigenstate positive height.

In a rough sea there is near height zero almost equal probability for finding water and air.

So if you act with an annihilation operator for a positive energy single fermion state or with a negative single fermion creation operator on a vacuum with interaction, then you obtain a state, in which the sum of the single fermion energies (ignoring the interaction) has been lowered.

The interacting vacuum is by definition the lowest energy state, when the interaction is included, but it is not the lowest energy state for the free fermion energy, so the free fermion energy can easily be lowered by some annihilation of a positive energy fermion or creation of a negative energy one.

This is analogous to that you could remove a droplet of water from a positive height position from a rough sea; or you could add a droplet in a negative height place, with some slight amount of luck only needed.

\section{A.1 The idea of the rough Dirac sea}

Really what we have in mind in the case of usual (particle) quantum field theory under the notation of the "rough Dirac sea" is just the true vacuum of the quantum field theory in the case of a strongly interacting theory. In a free quantum field theory one has a Dirac sea in which just all states with negative energy are filled while those with positive energy are empty so that

$$
\begin{array}{r}
a(\vec{p}, E>0)|0\rangle=0 \\
a^{+}(\vec{p}, E>0)|0\rangle \neq 0
\end{array}
$$


and

$$
\begin{aligned}
& a_{\text {anti }}(\vec{p}, E>0)|0\rangle=a^{+}(-\vec{p},-E<0)|0\rangle=0 \\
& a_{\text {anti }}^{+}(\vec{p}, E>0)|0\rangle=a(-\vec{p},-E<0)|0\rangle \neq 0 .
\end{aligned}
$$

But now if there are interactions the true vacuum get much more complicated and one could obtain it by a development of some state, e.g. the bare vacuum $|0\rangle$ through a long imaginary time so that the propagation operator becomes

$$
e^{-H_{t_{\text {large }}}}
$$

where $t_{\text {large }}$ is a very large time. Then we get the true vacuum

$$
|0\rangle_{\text {true }} \propto \lim _{t_{\text {large }}} \rightarrow \infty e^{-H_{t_{\text {large }}}}|0\rangle .
$$

If the interactions in Hamiltonian $H$ are strong the true vacuum is very much different from the bare one $|0\rangle$ Then it will be so that all operations with bare creation and annihilation operators $a^{+}(\vec{p}, E \gtrless 0)$ and $a(\vec{p}, E \gtrless 0)$ will give different from zero results when acting on $|0\rangle_{\text {true }}$.

I.e.

$$
\begin{array}{r}
a(\vec{p}, E>0)|0\rangle_{\text {true }} \neq 0 \\
a^{+}(\vec{p}, E>0)|0\rangle_{\text {true }} \neq 0 \\
a(\vec{p}, E<0)|0\rangle_{\text {true }} \neq 0 \\
a^{+}(\vec{p}, E<0)|0\rangle_{\text {true }} \neq 0 .
\end{array}
$$

Thus it is quite easy seemingly to add to the true vacuum negative energy. This is, however, only true when one thinks of the free approximation energy $H_{0}$, then one has

$$
\left\langle 0_{\text {true }}\left|a(\vec{p}, E<0) H_{0} a^{+}(\vec{p}, E<0)\right| 0_{\text {true }}\right\rangle<\left\langle\left. 0\right|_{\text {true }} H_{0} \mid 0\right\rangle_{\text {true }}
$$

But using true Hamiltonian $H$ instead of $H_{0}$ would mean that the true vacuum is the lowest eigenstate so that

$$
\langle b|H| b\rangle \geq\left\langle\left. 0\right|_{\text {ture }} H \mid 0\right\rangle_{\text {true }}
$$

for any state $|b\rangle$, also for

$$
|b\rangle=a^{+}(\vec{p}, E<0)|0\rangle_{\text {true }} .
$$

Even if we decide to make the here considered fermion be a Majorana fermion so that on the allowed states, the allowed subspace of the (second quantized) Hilbert space we have

$$
a_{\text {anti }}^{+}(\vec{p}, E>0) \stackrel{\text { effectively }}{=} a^{+}(\vec{p}, E>0) \text {. }
$$

We can still have this seeming - i.e. w.r.t. $H_{0}$ - addition of negative energy. 
In our present work we want to use the story of the "rough Dirac sea" for the objects ( $\sim$ bits of strings taken in the $X_{R}$ and $X_{L}$ instead of Thorns full $X$ ) - which in our scheme are essentially particles.

In our model we have, however, no interaction between objects and so a genuine true vacuum is not obvious to define. We so to speak lack $H$ just above. However, we can without really explaining it assume that there exist some especially selected — by God background state which we can identify with the true vacuum for the objects $|0\rangle_{\text {true (for obj) }}$ and then we should think of replacing the at first presented $\mid 0>$ as vacuum for the objects by the more complicated $|0\rangle_{\text {true (for obj) }}$

$$
|0\rangle \longrightarrow|0\rangle_{\text {true }}(\text { for obj) }
$$

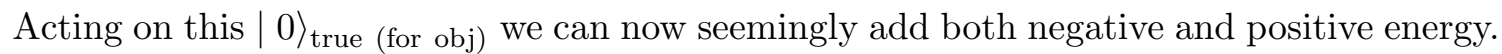
In this way one can on $|0\rangle_{\text {true (for obj) }}$ as background create states which have negative energy density along the cyclic chains, and they can be removed again by a positive energy preation operator series: e.g.

$$
\prod_{I} a^{+}\left(J^{\mu}(I)\right)|0\rangle_{\text {true (for obj) }}
$$

has compared $|0\rangle_{\text {true (for obj) }}$ itself a negative "bare" energy $\Sigma_{J^{0}}$ if the $J^{\mu}$ 's obey

$$
J^{0}(I)<0 .
$$

\section{B Use of rough Dirac sea analogy for our novel string field theory back- ground state}

Although we do not have any genuine interaction between the objects in our model, we shall nevertheless imagine that the "background state" on which we act with object-creation and object-annihilation operators is a complicated state, so that it is not the ground state for the sum of the single object states, so that it is not a problem to act with some annihilation or creation operator so as to add negative free energy, meaning make the sum of the object energies more negative.

Open Access. This article is distributed under the terms of the Creative Commons Attribution License (CC-BY 4.0), which permits any use, distribution and reproduction in any medium, provided the original author(s) and source are credited.

\section{References}

[1] H.B. Nielsen, A physical interpretation of the integrand of the n-point Veneziano model, Nordita preprint (1969).

[2] H.B. Nielsen, An almost physical interpretation of the integrand of the n-point Veneziano model, preprint at Niels Bohr Institute (1969).

[3] H.B. Nielsen, An almost physical interpretation of the integrand of the n-point Veneziano model, presented at the $15^{\text {th }}$ International Conference on High Energy Physics, August 26-September 4, Kiev, Russia (1970). 
[4] Y. Nambu, Quark Model and the factorization of the Veneziano amplitude, in Proceedings of the International Conference on Symmetries and Quark Models, June 18-20, 1969, R. Chand ed., Gordon and Breach, New York U.S.A. (1969), reprinted in Broken Symmetry Selected Papers, Y. Nambu et al. eds., World Scientific, Singapore (1995).

[5] Y. Nambu, Duality and hadrondynamics, Lecture notes prepared for Copenhagen summer school (1970), reprinted in Broken Symmetry Selected Papers, Y. Nambu et al. eds., World Scientific, Singapore (1995).

[6] L. Susskind, Structure of hadrons implied by duality, Phys. Rev. D 1 (1970) 1182.

[7] L. Susskind, Dual symmetric theory of hadrons, Nuovo Cim. A 69 (1970) 457.

[8] A. Cappeli et al., The birth of string theory, Cambridge University Press, Cambridge U.K. (2012).

[9] M.B. Green, J.H. Schwarz and E. Witten, Superstring theory, Cambridge University Press, Cambridge U.K. (1987).

[10] J. Polchinski, String theory, Cambridge University Press, Cambridge U.K. (1998).

[11] W. Taylor and B. Zwiebach, D-branes, tachyons and string field theory, hep-th/0311017 [INSPIRE].

[12] K. Ohmori, A review on tachyon condensation in open string field theories, hep-th/0102085 [INSPIRE].

[13] M. Kaku and K. Kikkawa, The field theory of relativistic strings. Part 1. Trees, Phys. Rev. D 10 (1974) 1110 [INSPIRE].

[14] M. Kaku and K. Kikkawa, The field theory of relativistic strings. Part 2. Loops and pomerons, Phys. Rev. D 10 (1974) 1823 [INSPIRE].

[15] S. Mandelstam, Interacting string picture of dual resonance models, Nucl. Phys. B 64 (1973) 205 [INSPIRE].

[16] E. Cremmer and J.-L. Gervais, Infinite component field theory of interacting relativistic strings and dual theory, Nucl. Phys. B 90 (1975) 410 [INSPIRE].

[17] H. Hata, K. Itoh, T. Kugo, H. Kunitomo and K. Ogawa, Covariant string field theory, Phys. Rev. D 34 (1986) 2360 [INSPIRE].

[18] H. Hata, K. Itoh, T. Kugo, H. Kunitomo and K. Ogawa, Covariant string field theory. 2., Phys. Rev. D 35 (1987) 1318 [INSPIRE].

[19] H. Hata, K. Itoh, T. Kugo, H. Kunitomo and K. Ogawa, Loop amplitudes in covariant string field theory, Phys. Rev. D 35 (1987) 1356 [INSPIRE].

[20] M.B. Green and J.H. Schwarz, Superstring interactions, Nucl. Phys. B 218 (1983) 43 [INSPIRE].

[21] M.B. Green, J.H. Schwarz and L. Brink, Superfield theory of type II superstrings, Nucl. Phys. B 219 (1983) 437 [INSPIRE].

[22] M.B. Green, and J.H. Schwarz, Superstring field theory, Nucl. Phys. B 243 (1984) 437.

[23] J. Greensite and F.R. Klinkhamer, New interactions for superstrings, Nucl. Phys. B 281 (1987) 269 [INSPIRE].

[24] J. Greensite and F.R. Klinkhamer, Contact interactions in closed superstring field theory, Nucl. Phys. B 291 (1987) 557 [nSPIRE]. 
[25] J. Greensite and F.R. Klinkhamer, Superstring amplitudes and contact interactions, Nucl. Phys. B 304 (1988) 108 [INSPIRE].

[26] M.B. Green and N. Seiberg, Contact interactions in superstring theory, Nucl. Phys. B 299 (1988) 559 [INSPIRE].

[27] S.-J. Sin, Geometry of super light cone diagrams and Lorentz invariance of light cone string field theory. 2. Closed Neveu-Schwarz string, Nucl. Phys. B 313 (1989) 165 [inSPIRE].

[28] E. Witten, Noncommutative geometry and string field theory, Nucl. Phys. B 268 (1986) 253 [INSPIRE].

[29] M. Saadi and B. Zwiebach, Closed string field theory from polyhedra, Annals Phys. 192 (1989) 213 [INSPIRE].

[30] T. Kugo, H. Kunitomo and K. Suehiro, Nonpolynomial closed string field theory, Phys. Lett. B 226 (1989) 48 [INSPIRE].

[31] T. Kugo and K. Suehiro, Nonpolynomial closed string field theory: action and its gauge invariance, Nucl. Phys. B 337 (1990) 434 [INSPIRE].

[32] B. Zwiebach, Closed string field theory: quantum action and the B-V master equation, Nucl. Phys. B 390 (1993) 33 [hep-th/9206084] [InSPIRE].

[33] E. Witten, Interacting field theory of open superstrings, Nucl. Phys. B 276 (1986) 291 [INSPIRE].

[34] I.Ya. Arefeva and P.B. Medvedev, Anomalies in Witten's field theory of the NSR string, Phys. Lett. B 212 (1988) 299 [inSPIRE].

[35] C. Wendt, Scattering amplitudes and contact interactions in Witten's superstring field theory, Nucl. Phys. B 314 (1989) 209 [InSPIRE].

[36] N. Berkovits, SuperPoincaré invariant superstring field theory, Nucl. Phys. B 450 (1995) 90 [Erratum ibid. B 459 (1996) 439] [hep-th/9503099] [INSPIRE].

[37] I.Ya. Arefeva, P.B. Medvedev and A.P. Zubarev, New representation for string field solves the consistency problem for open superstring field theory, Nucl. Phys. B 341 (1990) 464 [INSPIRE].

[38] C.R. Preitschopf, C.B. Thorn and S.A. Yost, Superstring field theory, Nucl. Phys. B 337 (1990) 363 [INSPIRE].

[39] S. Mandelstam, Interacting string picture of dual resonance models, Nucl. Phys. B 64 (1973) 205 [INSPIRE].

[40] S. Mandelstam, Interacting string picture of the Neveu-Schwarz-Ramond model, Nucl. Phys. B 69 (1974) 77 [INSPIRE].

[41] P. Goddard, J. Goldstone, C. Rebbi and C.B. Thorn, Quantum dynamics of a massless relativistic string, Nucl. Phys. B 56 (1973) 109 [INSPIRE].

[42] H.B. Nielsen and M. Ninomiya, A new type of string field theory, AIP Conf. Proc. 607 (2001) 185 [hep-th/0111240] [INSPIRE].

[43] H.B. Nielsen and M. Ninomiya, An idea of new string field theory - Liberating right and left movers, in the proceedings of the $14^{\text {th }}$ Workshop, What Comes Beyond the Standard Models, July 11-21, Bled, Slovenia (2011), arXiv:1112.0542. 
[44] H.B. Nielsen and M. Ninomiya, A novel string field theory solving string theory by liberating left and right movers, JHEP 05 (2014) 026 [arXiv: 1211.1454].

[45] R. Giles and C.B. Thorn, A lattice approach to string theory, Phys. Rev. D 16 (1977) 366 [INSPIRE].

[46] C.B. Thorn, On the derivation of dual models from field theory, Phys. Lett. B 70 (1977) 85.

[47] C.B. Thorn, On the derivation of dual models from field theory. 2., Phys. Rev. D 17 (1978) 1073 [INSPIRE].

[48] C.B. Thorn, Reformulating string theory with the $1 / N$ expansion, in the proceedings of Sakharov Memorial Lectures in Physics, May 21-31, Moscow, Russia (1991), hep-th/9405069 [INSPIRE].

[49] O. Bergman and C.B. Thorn, String bit models for superstring, Phys. Rev. D 52 (1995) 5980 [hep-th/9506125] [INSPIRE].

[50] C.B. Thorn, Space from string bits, JHEP 11 (2014) 110 [arXiv:1407.8144] [InSPIRE].

[51] G. Veneziano, Construction of a crossing-symmetric, Regge behaved amplitude for linearly rising trajectories, Nuovo Cim. A 57 (1968) 190.

[52] K.G. Wilson, Confinement of quarks, Phys. Rev. D 10 (1974) 2445 [InSPIRE].

[53] H.B. Nielsen and M. Ninomiya, Absence of neutrinos on a lattice I - Proof by homotopy theory (1981).

[54] Z. Koba and H.B. Nielsen, Manifestly corssing-invariant parameterization of n-meson amplitude, Nucl. Phys. B 12 (1969) 243. 